\title{
Requirement of co-factors for the ligand-mediated activity of the insect ecdysteroid receptor in yeast
}

\author{
H T Tran ${ }^{1}$, S Shaaban ${ }^{1}$, H B Askari ${ }^{1}$, P G Walfish ${ }^{2}$, A S Raikhel ${ }^{3}$ \\ and $\mathbf{T} \mathbf{R}$ Butt $^{1}$ \\ ${ }^{1}$ LifeSensors Inc., 271 Great Valley Parkway, Malvern, Pennsylvania 19355, USA \\ ${ }^{2}$ Gene Expression Laboratory, Division of Endocrinology and Metabolism, University of Toronto \\ Medical School, Toronto, Ontario M5 G1X5, Canada \\ ${ }^{3}$ Department of Entomology and Program in Cell and Molecular Biology, Michigan State University, \\ East Lansing, Michigan 48824, USA
}

(Requests for offprints should be addressed to T R Butt; Email: butt@lifesensors.com)

\begin{abstract}
In insects, a steroid hormone 20-hydroxyecdysone has an important role in regulating critical events such as development and reproduction. The action of 20-hydroxyecdysone is mediated by its binding to the ecdysteroid receptor $(\mathrm{EcR})$, which requires a heterodimeric partner, ultraspiracle protein (USP), a homologue of the retinoid $\mathrm{X}$ receptor (RXR). The EcR-USP heterodimer represents a functional receptor complex capable of initiating transcription of early genes. Our goal was to establish a ligand-dependent transactivation system in yeast utilizing an insect EcR-USP heterodimer. This has been achieved using mosquito Aedes aegypti AaEcR-USP. Expression of AaEcR alone, but not USP, resulted in constitutive transcription of the ecdysone reporter gene coupled with the Drosophila heat shock protein-27 ecdysone response elements. Removal of the N-terminal A/B domain of AaEcR abolished its constitutive transcription. Constitutive transcription was also eliminated in the presence of its heterodimeric partner, $\mathrm{AaUSPa}, \mathrm{AaUSPb}$ or
\end{abstract}

mammalian RXR. This suggests that the A/B domain is essential for the EcR ligand-independent transactivation and its interaction with the yeast transcription complex. A ligand-mediated transactivation of $\mathrm{Aa}(\Delta \mathrm{A} / \mathrm{B}) \mathrm{EcR}-\mathrm{USP}$ or $\mathrm{Aa}(\Delta \mathrm{A} / \mathrm{B}) \mathrm{EcR}-$ $\mathrm{RXR}$ heterodimers in response to an ecdysteroid agonist RH-5992 was observed only in the presence of GRIP1, a mouse co-activator. In the presence of a co-repressor, SMRT, $\mathrm{Aa}(\Delta \mathrm{A} / \mathrm{B}) \mathrm{EcR}-\mathrm{USP}$ heterodimer exhibited a ligand-dependent repression activity. In addition, ligand-dependent transactivation systems for spruce budworm and fruit fly ecdysone receptors were also reported. This is the first report establishing the requirements of cofactors for a highly efficient ligand-dependent function of the insect EcR-USP in yeast. These findings open a way to study insect EcR-USP structure and function and to identify ligands that are specific for a certain group of insects, such as mosquitoes.

Fournal of Molecular Endocrinology (2001) 27, 191-209

\section{INTRODUCTION}

Ecdysteroids, the arthropod steroid hormones, have a crucial role in coordinating molting, metamorphosis and reproduction (Riddiford 1993, Thummel 1995). The action of ecdysteroids is mediated by the heterodimeric complex, consisting of two nuclear receptors, ecdysteroid receptor itself $(\mathrm{EcR})$ and the insect homologue of the retinoid X receptor (RXR), ultraspiracle (USP). Upon binding the ligand, the EcR-USP heterodimer binds to a DNA ecdysone response element (EcRE) that is located in the promoter region of a series of ecdysteroidresponsive genes, and thereafter triggers the expression of a cascade of genes involved in regulating some key developmental events in insects. The formation of the EcR-USP heterodimer is required for both DNA and ligand binding (Yao et al. 1993, Kapitskaya et al. 1996). In addition to Drosophila melanogaster EcR (Koelle et al. 1991), several insect ecdysteroid receptors have now been cloned (Imhof et al. 1993, Cho et al. 1995, Fujiwara et al. 1995, Kothapalli et al. 1995, Swevers et al. 1995, Jindra et al. 1997, Verras et al. 1999). 
A

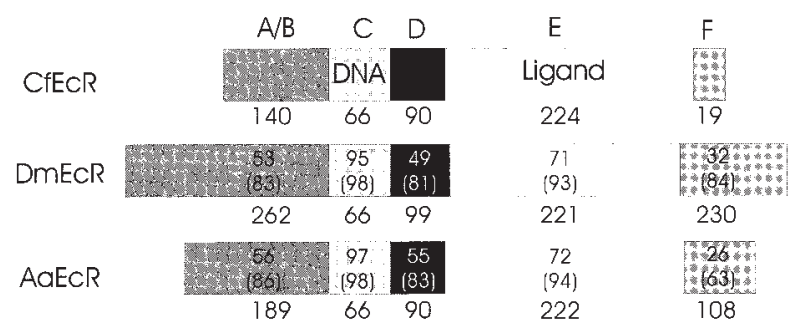

$\mathbf{B}$

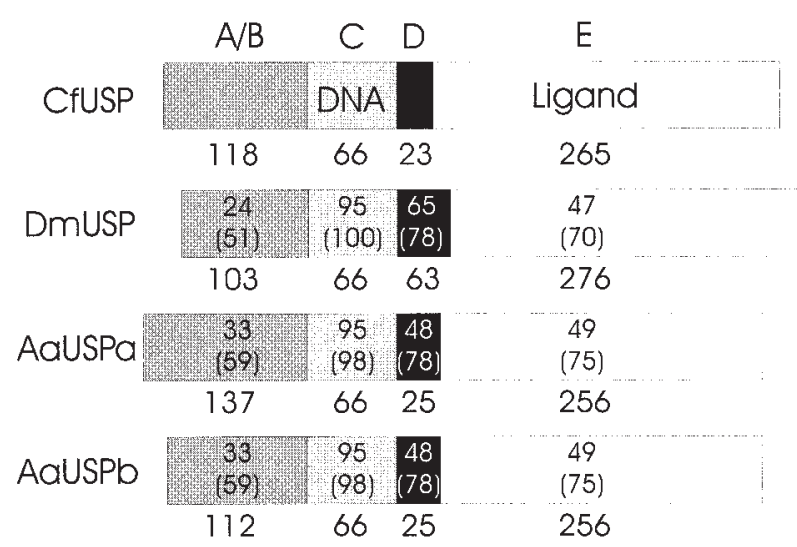

FIGURE 1. Domain comparison of insect EcR (A) and USP (B) receptors. Percent amino acid identity (top number) and percent amino acid similarity (bottom number) are indicated in each corresponding domain in comparison with CfEcR and CfUSP respectively. The number below each domain is its length in amino acids. (A) Alignment of CfEcR (Kothapalli et al. 1995), DmEcR-B1 (Koelle et al. 1991) and AaEcR (Cho et al. 1995). (B) Alignment of CfUSP (Perera et al. 1998), DmUSP (Henrich et al. 1990), AaUSPa and AaUSPb (Kapitskaya et al. 1996).

Likewise, in addition to D. melanogaster USP (Henrich et al. 1990, Oro et al. 1990, Shea et al. 1990), the heterodimer partner of EcR has also been cloned from several insects (Tzertzinis et al. 1994, Kapitskaya et al. 1996, Jindra et al. 1997, Perera et al. 1998).

The pleiotropic effects of ecdysteroids are reflected by the existence of several isoforms of both $\mathrm{EcR}$ and USP, which differ in their A/B domains. EcR isoforms were first identified in Drosophila (Talbot et al. 1993) and then in other insects (Fujiwara et al. 1995, Swevers et al. 1995, Kamimura et al. 1996, 1997, Jindra et al. 1996, Perera et al. 1998), including the mosquito Aedes aegypti (S F Wang, C Li \& A S Raikhel, unpublished data). Unlike Drosophila USP, for which a single form of mRNA has been identified (Henrich et al. 1990, Oro et al. 1990, Shea et al. 1990), the mosquito A. aegypti (Kapitskaya et al. 1996, Wang et al. 2000b) and the tobacco hornworm Manduca sexta (Jindra et al. 1997, Lan et al. 1999) have been reported to have two isoforms.

The EcR partner can form a functional heterodimer with the USP protein from other insects, and even with mammalian RXR (Thomas et al. 1993, Yao et al. 1993, Verras et al. 1999, Wang et al. 1998). For example, Mediterranean fruit fly Ceratitis capitata ecdysone receptor $(\mathrm{CcEcR})$ binds specifically to the $D$. melanogaster heat shock protein-27 (hsp27) EcRE as a heterodimer with DmUSP (Verras et al. 1999). For the mosquito, the EcR subunit has been shown to determine specificity of ligand binding (Wang et al. 2000a). Furthermore, Drosophila EcR has been shown to function with mammalian heterodimeric partners RXR (Yao et al. 1992, Hatzivassiliou et al. 1997).

EcRs or USPs have high homology in the corresponding DNA binding domains, but their $\mathrm{A} / \mathrm{B}$ domains, which contain putative activation function (AF)-1, and ligand binding domains (LBDs), which contain putative AF-2 regions, share relatively low homology (Fig. 1; Kothapalli et al. 1995, Perera et al. 1998). Disrupting the ecdysteroid regulation cascade can be exploited as a new way for disruption of insect development. The low level of homology in the LBDs of EcR could be exploited to develop ligands that are specific for defined EcR. Subsequently, these compounds allow the disruption of the molting process in a pest-specific manner. The non-steroidal ecdysteroid agonist, RH-5992 that mimicks the action of ecdysteroid was used as a pesticide targeting lepidopteran larvae (Retnakaran et al. 1995). This compound mainly targets lepidopteran larvae, and is generally devoid of toxicity in non-lepidopteran species, including a wide range of important predators and parasites (Dhadialla et al. 1998).

A ligand-mediated transactivation system of EcR-USP and EcR-RXR has been developed in mammalian and insect tissue cultures (Thomas et al. 1993, Lan \& Riddiford 1997). However, as there are many nuclear receptors expressed in mammalian and insect cells, the effect of ligand on the heterodimer might be compromised as a result 
of the influence of other nuclear receptors. For example, in mosquito $A$. aegypti, expression of AHR38 can block heterodimeric formation of AaEcR-AaUSP and its activity as AHR38 competes for AaUSP (Zhu et al. 2000). Expression of the $M$. sexta USP-2 isoform can eliminate activation of MHR3 induced by the MsEcR-B1-USP-1 complex, as MsUSP-2 is competing with MsUSP-1 for MsEcR-B1 (Lan et al. 1999). In contrast to insect cells, yeast does not contain either nuclear receptors or any known homologues of mammalian co-activators. However, because of conservative general transcription machinery in eukaryotes, transactivation systems of mammalian nuclear receptors have been successfully reconstructed in yeast (see Butt \& Walfish 1996). These systems have been used for structural analysis of various nuclear receptors, validation, and screening of pharmaceuticals (Butt \& Walfish 1996, Butt \& Chen 1999).

Several groups of investigators have tried in vain to reconstruct ligand-mediated transactivation of EcR-USP in yeast, because of lack of activity or high constitutive transcription activity (Dela Cruz \& Mak 1997, Dela Cruz et al. 2000). Recently, we have reported the construction of a liganddependent transactivation system in yeast for spruce budworm Choristoneura fumiferana ecdysone receptor (Tran et al. 2001). This work describes the first example of yellow fever mosquito EcR-USP ligand-dependent transactivation system that responds to ecdysteroids and their analogue RH-5992, and its interaction with different co-activators. In addition to this, the ligand-dependent transactivation of $D$. melanogaster EcR-USP is also presented. The availability of a ligand-dependent transactivation systems for insect EcRs would provide an effective means to discover new chemicals, and validate and improve potential insecticidal candidates. Furthermore, this system could also provide a tool for structural and functional analysis of EcR with ecdysteroids, and with its partner USPs and other co-activators.

In this study, we report the reconstitution of a ligand-mediated transactivation system in yeast using the yellow fever mosquito $A$. aegypti EcR and USP. We have demonstrated that expression of the full-length of AaEcR alone can induce ligandindependent constitutive transcription of the reporter gene coupled with a promoter harboring the natural Drosophila hsp27 EcREs. Removal of the A/B domain of the AaEcR abolished constitutive transactivation activity of $\mathrm{EcR}$, suggesting the important role of the $\mathrm{A} / \mathrm{B}$ domain in ligandindependent transactivation. Co-expression of the $\mathrm{A} / \mathrm{B}$ domain truncated $\mathrm{EcR}(\mathrm{Aa} \Delta \mathrm{EcR})$ with its heterodimer partners $\mathrm{AaUSPa}, \mathrm{AaUSPb}$ or mam- malian USP homolog RXRs failed to restore transactivation activity even in the presence of ligands. We tested the role of the following co-activators or co-repressors: GRIP1 (Hong et al. 1996), RIP140 (Cavailles et al. 1995), Drosophila CBP (Akimaru et al. 1997), mouse CBP (Chrivia et al. 1993), SMRT (Chen et al. 1996), SRC-1 (Onate et al. 1995), and N-coR (Horlein et al. 1995) in a ligand-dependent transactivation of insect EcRUSP complex. A ligand-mediated response was observed only when GRIP1, a mouse co-activator, was added to $\mathrm{Aa} \Delta \mathrm{EcR}-\mathrm{RXR}$ or $\mathrm{Aa} \Delta \mathrm{EcR}-\mathrm{USP}$ complexes. In contrast to the effect of GRIP1, the co-repressor SMRT exhibited ligand-dependent repression of constitutive activity. Different USPs such as mosquito USPa, USPb, USPs from $C$. fumiferana or D. melanogaster, or mammalian RXRs distinctively modulate the response of $\mathrm{AaEcR}$ to ligands. In addition to AaEcR-USP, we also reported ligand-dependent transactivation activity of spruce budworm C. fumiferana and D. melanogaster EcR-USP complexes in yeast. The transactivation responses to different compounds of these insect EcR-USPs in combination with series of cofactors are different, suggesting that insect EcR-USP activity is regulated by both ligands and co-activators/co-repressors. These findings open a way to study the insect EcR-USP complex and for discovering ligands for insect EcR and USP.

\section{MATERIALS AND METHODS}

\section{Media, strains, plasmids and ligands}

Standard yeast and Escherichia coli media have been prepared as described previously (Sherman et al. 1986, Sambrook et al. 1989). A yeast strain Y4727 snq2::4 pdr5::kanMX mutant in the ABC transporter pathway was used as a host for transformation. The strain was derived from the yeast strain Y4727: Mata his3-4200 leu2- $\Delta 0$ lys $2-\Delta 0$ met $5-\Delta 0$ trp1- 463 ura3- $\Delta 0$ (gift from Dr Jeff Boeke, Johns Hopkins University) by deleting the whole open reading frame of the SNQ2 and the PDR5 genes using the PCR-transformation technique as described previously (Storici et al. 1999). Yeast transformation was performed according to procedures described by Gietz et al. (1992). Yeast transformants with plasmids were maintained in correspondent drop-out selective media. Multicopy yeast-E.coli shuttle plasmids containing the fulllength and $\mathrm{A} / \mathrm{B}$-domain-deleted receptor $\mathrm{AaEcR}$ and full-length $\mathrm{AaUSPa}$ and $\mathrm{AaUSPb}$ plasmids in addition to A/B-domain deleted receptor AaUSP (Aa $\Delta$ USP) were constructed (see below). The 
mammalian RXR $\alpha, \operatorname{RXR} \beta$ or RXR $\gamma$ subtypes (Allegretto et al. 1993) were expressed in $2 \mu \mathrm{m}$ multicopy plasmids under regulation of a CUP1 promoter with a LEU2 selective marker. The series of co-activators and co-repressors such as GRIP1 (Hong et al. 1996), RIP140 (Cavailles et al. 1995), Drosophila CBP (Akimaru et al. 1997), mouse CBP (Chrivia et al. 1993), SMRT (Chen et al. 1996), SRC-1 (Onate et al. 1995), and N-coR (Horlein et al. 1995) were also expressed as yeast $2 \mu \mathrm{m}$ multicopy plasmids with a HIS3 selective marker. Construction of these plasmids is described below. The yeast expression plasmid with GRIP1 (Hong et al. 1996) was constructed as follows. The NsiI-BamHI (GRIP1 gene with ADH1 promoter) fragment from the pGRIP812 (Walfish et al. 1997) was blunt-ended and cloned into the PvuII site of the pRS423 (Sikorski \& Hieter 1989). The $\beta$-galactosidase reporter gene containing six copies of natural ecdysone response element (hsp27-EcRE) was also constructed (see below).

Ecdysteroid non-steroidal agonists RH-1, RH-2, RH-3, RH-4, RH-5, RH-6, RH-0345, RH-2485 and RH-5992 are derivatives of the original compound RH-5849 (Dhadialla et al. 1998). The structural formulae of the compounds RH-0345, RH-2485, RH-5849 and RH-5992 were published previously (Dhadialla et al. 1998). Muristerone A was purchased from Sigma. All ligands were dissolved in DMSO with a stock solution concentration of $500 \mu \mathrm{M}$. The final ligand concentration in the yeast cell culture was $10 \mu \mathrm{M}$.

\section{Plasmid constructions}

The plasmid pBRSS-6xEcRE-lacZ is a reporter construct carrying six copies of natural EcRE from the D. melanogaster heat shock protein-27 (hsp27) gene (Riddihough \& Pelham 1987). The response elements are located in the upstream of the iso-1-cytochrome $\mathrm{C}$ (CYC1) promoter that is coupled with $E$. coli $\beta$-galactosidase gene (lacZ). This yeast-E. coli multicopy shuttle plasmid contains URA3 as a yeast transformation marker. Full-length or A/B domain-deleted versions of $\mathrm{AaEcR}$ were fused at the $\mathrm{N}$-terminal in frame with human ubiquitin which was under CUP1 promoter (plasmids YEp-AaEcR and $\mathrm{YEp}-\mathrm{Aa} \Delta \mathrm{EcR}$, respectively). The $2 \mu \mathrm{m}$ yeast expression plasmids YEp$\mathrm{AaEcR}$ and $\mathrm{YEp}-\mathrm{Aa} \Delta \mathrm{EcR}$ have been constructed, based on the plasmid YEpE12 (Graumann et al. 1996), which contains the TRP1 as a yeast selective marker, and ubiquitin-fused human estrogen receptor (ER) under CUP1 promoter. The YEpE12 plasmid was previously used for reconstruction of ligand-dependent transactivation of $\mathrm{ER}$ in yeast
(Graumann et al. 1996). The following primers AaEcR-SalI and AaEcR-MluI were used for amplification of the full-length of AaEcR from cDNA (Cho et al. 1995). With AaEcR-SalI: 5'GGAGTCGACCTTACATCTTGTCTTAAG ACTAAGAGGTGGTatgatgaaaagaagatggtcc - 3' (upper case indicates the nucleotide sequence belonging to human ubiquitin, the SalI site available in the ubiquitin is underlined, and lower case denotes the sequence belonging to $\mathrm{AaEcR}$, starting from ATG). With AaEcR-MluI: 5'-AAGGAC GCGTtgaacagaatgtcgtccgct-3' (lower case indicates the nucleotide sequences belonging to $3^{\prime}$ terminal of the AaEcR, and the MluI restriction site is underlined). For amplification of the $\mathrm{AaEcR}$ with A/B domain deletion (Fig. 1) the following primers were used: AaEcR-MluI (above) and $\mathrm{Aa} \Delta \mathrm{EcR}-\mathrm{SalI}$ : 5'-AGGAGTCGACCTTACATCTTGTCTTA AGACTAAGAGGTGGTatgcggcagcaggaggaactg tgtctg-3' (upper case indicates the nucleotide sequence belonging to human ubiquitin, the SalI site available in the ubiquitin is underlined, and lower case denotes the sequence belonging to the DNA binding domain of the AaEcR starting from amino acid RQQEELCLV). After protein translation and ubiquitin cleavage, the arginine ' $R$ ' in the protein $\mathrm{N}$-terminal would be exposed. That is proposed to be a signal for short-life protein (Bachmair et al. 1986), therefore, an additional methionine is added before the RQQEELCVL, to stabilize the protein. The DNA fragments were amplified in 30 cycles $\left(96{ }^{\circ} \mathrm{C}\right.$ for $30 \mathrm{~s}, 54^{\circ} \mathrm{C}$ for $1 \mathrm{~min}$, and $72{ }^{\circ} \mathrm{C}$ for $3 \mathrm{~min}$ ) using high replication fidelity Deep Vent Polymerase (New England Biolabs). The PCR products for both full-length $\mathrm{AaEcR}$ and $\mathrm{Aa} \Delta \mathrm{EcR}$ were digested with SalI and MluI and subsequently subcloned into the SalI and MluI sites of the plasmid YEpE12 (Graumann et al. 1996).

Mosquito $A$. aegypti expresses two forms of USP (A and $B$ isoforms) that differ only in the N-terminal region in the $\mathrm{A} / \mathrm{B}$ domain (Fig. 1; Kapitskaya et al. 1996). The yeast expression vectors for A and B isoforms (pRS425-AaUSPa and pRS425-AaUSPb) and for AaUSP with deletion of the A/B domain (pRS425-Aa $\Delta$ USP) were constructed as follows. The A and B isoforms of USP and $\triangle$ USP were amplified from corresponding cDNAs using the following pairs of primers: AaUSPa-5' and AaUSP-3'; AaUSPb-5' and AaUSP-3'; and Aa $\triangle{ }^{\prime} \mathrm{USP}_{-} 5^{\prime}$ and AaUSP-3', respectively. AaUSPa-5': 5'-AGGAGTCGACCTT ACATCTTGTCTTAAGACTAAGAGGTGGT atgctgaagaaggaaaaacc-3'; AaUSPb-5': 5'-AGGA GTCGACCTTACATCTTGTCTTAAGACTAA GAGGTGGTatggatcccagcgatcgagg-3; Aa $\Delta$ USP-5': 5'-AGGAGTCGACCTTACATCTTGTCTTAAG 
ACTAAGAGGTGGTatgtatccgccaaatcatccgctcagc-3' (upper case indicates the nucleotide ubiquitin sequence, lower case denotes the nucleotide sequence of $5^{\prime}$-terminal of the AaUSPa and $\mathrm{AaUSPb}$ isoforms, starting from the first ATG codon, or starting from the DNA binding domain of the USP (MYPPNH) respectively, and the ubiquitin SalI site is underlined); and AaUSP-3': 5'AAGGACGCGTccacaagttgcttgttctagg- $3^{\prime}$ (the MluI site is underlined, and lower case indicates the nucleotide sequence of the $3^{\prime}$ terminal of AaUSP cDNA. This primer is common for both the USPa and USPb nucleotide sequences). The PCR products were purified and digested with SalI and MluI and subsequently subcloned into the SalI-MluI sites of the yeast expression vector with the LEU2 marker pRS425-ER $\alpha$. (The plasmid pRS425-ER $\alpha$ was constructed as follows: the BamHI-PmlI CUP1p$\mathrm{ER} \alpha-\mathrm{cyc}_{\text {ter }}$ fragment from the YEpE12 plasmid (Graumann et al. 1996) was blunt-ended and then ligated into the PvuII site of pRS425 (Sikorski \& Hieter 1989).)

The A/B domain-deleted version of $\mathrm{CfEcR}$ was fused at the $\mathrm{N}$-terminal in frame with human ubiquitin which is also under CUP1 promoter (plasmids YEp-Cf $\Delta \mathrm{EcR}$ ). The multicopy yeast expression plasmid YEpCf $\Delta \mathrm{EcR}$ has been constructed based on the plasmid YEpE12 (Graumann et al. 1996) that contains the TRP1 as a yeast selective marker, and ubiquitin-fused human ER under CUP1 promoter. The following pair of primers, Cf $\Delta$ EcR-Sal I and CfEcR-Sac I, were used for amplification of the A/B domain-deleted CfEcR from the cDNA clone (Kothapalli et al. 1995). Cf $\Delta$ EcR-Sal I: 5'-GGAGTCGACCTTACATCT TGTCTTAAGACTAAGAGGTGGTatgcggcagca ggaggaactgtgtctg-3' (upper case indicates the nucleotide sequence belonging to human ubiquitin, the Sal I site available in the ubiquitin is underlined, and lower case denotes the sequence belonging to the DNA binding domain of the CfEcR starting from amino acid RQQEELCLV); CfEcR-SacI primer: 5'-AAGGGAGCTCtaatctccc gcgcattc- $3^{\prime}$ (lower case indicates the nucleotide sequences belonging to the $3^{\prime}$ terminal of the $\mathrm{CfEcR}$, and the SacI restriction site is underlined). The A/B domain-deleted EcR $(\Delta \mathrm{EcR})$ started from the beginning of the DNA binding domain with amino acid sequence RQQEELCLV. After protein translation and ubiquitin cleavage, the arginine residue ' $R$ ' in the protein N-terminal that is a proposed signal for short life protein would be exposed (Bachmair et al. 1986), therefore an additional methionine was added before the RQQEELCVL to stabilize the protein. The DNA fragments were amplified in 30 cycles $\left(96{ }^{\circ} \mathrm{C}\right.$ for
$30 \mathrm{~s}, 54{ }^{\circ} \mathrm{C}$ for $1 \mathrm{~min}$, and $72{ }^{\circ} \mathrm{C}$ for $3 \mathrm{~min}$ ) using high replication fidelity Deep Vent Polymerase (New England Biolabs). The PCR products for $\mathrm{Cf} \Delta \mathrm{EcR}$ were digested with SalI and SacI and subsequently recloned into the SalI and SacI sites of the plasmid YEpE12. The yeast expression vectors for spruce budworm $C$. fumiferana USPCfUSP and CfUSP with deletion of the A/B domain (pRS425-CfUSP and pRS425-Cf $\Delta$ USP respectively) were constructed similarly to the vectors with mosquito USP. Initially, the fulllength CfUSP or CfAUSP was amplified from a cDNA clone (Fig. 1, Kothapalli et al. 1995) using the following pairs of primers: CfUSP-5' and CfUSP- ${ }^{\prime}$ and CfAUSP-5' and CfUSP-3', respectively. CfUSP-5': 5'-AGGAGTCGACCTTACAT CTTGTCTTAAGACTAAGAGGTGGTatgtcaag tgtggcgaag-3'; CfDUSP-5': 5'-AGGAGTCGACC TTACATCTTGTCTTAAGACTAAGAGGTGG Tqatgtacccgcctaatcaccccctgagt-3' (upper case letters correspond to the nucleotide sequence of ubiquitin, lower case letters denote the nucleotide sequence corresponding to the $5^{\prime}$-terminus of the CfUSP starting from the ATG or from the DNA binding domain of the CfUSP respectively, and the ubiquitin SalI site is underlined); CfUSP-3': 5'-CCTTCCATGGgaatg tcaataatgcccgtg- $3^{\prime}$ (the NcoI site is underlined, and the lower case letters indicate the nucleotide sequence of the $3^{\prime}$ terminus of CfUSP cDNA). The PCR products were purified and digested with SalI and NcoI and subsequently subcloned into the SalI-NcoI sites of a yeast expression vector containing a LEU2 selective marker, pRS425-ER $\alpha$.

The DmUSP cDNA clone was used as a source for the plasmid construct. The N-terminal coding sequence of the DmUSP (Henrich et al. 1990, Oro et al. 1990) was amplified using two primers: DmUSP-AfII primer (5'-TTGTCTTAAGAC TAAGAGGTGGTatggacaactgcgaccagg-3') and DmUSP-NcoI-primer (5'-agcaggtggaccatggacatgg$\left.3^{\prime}\right)$ (upper case letters indicate the sequence corresponding to ubiquitin, lower case letters indicate sequences corresponding to DmUSP, and the AfII and NcoI sites are underlined). The PCR fragment was digested with AflI-NcoI and subcloned into the AflI and NcoI sites of the plasmid YEpUbOCT1. The resulting plasmid contains the DmUSP cDNA sequence fused $5^{\prime}$ in frame with ubiquitin. Next, the NcoI-AflII II fragment of the DmUSP cDNA (Henrich et al. 1990, Oro et al. 1990) was cloned into the NcoI-Acc65I of the intermediate plasmid obtained above (both AfII and Acc65I sites were blunt-ended using Klenow DNA polymerase). The resulting plasmid, YEpDmUSP encodes the full-length of the DmUSP amino acid sequence fused in frame with ubiquitin 
sequences, expression of each being driven by the CUP1 promoter. The TRP1 selective marker is included to facilitate selection of transformants. The DmUSP cDNA sequence was also shuffled to another plasmid pRS425-ER $\alpha$, containing the LEU2 with human ERa fused to ubiquitin under CUP1 promoter via the following the procedure. The SalI-MluI fragment containing a portion of ubiquitin and the entire coding region of the USP sequence from YEp-DmUSP was cloned into the SalI-MluI site of pRS425-ER $\alpha$. The resultant plasmid, pRS425-DmUSP, contains a LEU2 selective marker. The USP sequence is fused in frame with ubiquitin and USP expression is driven by the CUP1 promoter. The yeast expression vector encoding the DmUSP with an A/B domain deletion (Dm $\Delta$ USP, Fig. 1) was constructed as follows. The pRS425-DmUSP plasmid containing the full coding sequence of the DmUSP was used to amplify an N-terminally truncated DmUSP. First, the A/B domain deleted DmUSP was amplified using two primers: Dm $\Delta$ USP-5': (5'-AGGAGTCGACCTT ACATCTTGTCTTAAGACTAAGAGGTGGTat gtatccgcctaaccatccgctgagc- $3^{\prime}$; upper case letters indicate the nucleotide ubiquitin sequence, lower case letters denote the nucleotide sequence of 5 -terminus of DmUSP starting from YPPNH, and the SalI site is underlined) and DmUSP-3' (5'-AAGG ACGCGTcttttcggttagagcggatg-3'; (MluI site underlined, and lower case letters indicate the nucleotide sequence of the $3^{\prime}$ terminus of DmUSP cDNA). The PCR products were purified and digested with SalI and MluI and subsequently subcloned into SalIMluI sites of the yeast expression vector with LEU2 marker, pRS425-ER $\alpha$ as described above. Similar to the plasmid YEpCf $\Delta \mathrm{EcR}$, the plasmid $\mathrm{YEpDm} \Delta \mathrm{NEcR}$ is the yeast expression plasmid with TRP1 marker, encoding the $\mathrm{N}$-terminal truncated $D$. melanogaster EcR B-1 ecdysone receptor. The first 220 amino acids up to the sequence VNSSISS have been deleted and the resulting sequence is inserted into the yeast expression vector to produce a ubiquitin fusion protein under the control of a CUP1 promoter.

\section{$\beta$-Galactosidase activity assay}

A method to measure a $\beta$-galactosidase activity was developed to estimate a potency of compounds and to be simple enough to be applied in highthroughput screening. The transformed yeast cells with plasmids were grown overnight in selective liquid media at $30{ }^{\circ} \mathrm{C}$ and diluted in pre-warmed selective liquid media to $0 \cdot 1$ at an optical density of $600 \mathrm{~nm}\left(\mathrm{OD}_{600} ; \mathrm{OD}_{\text {culture }}\right) . \mathrm{CuSO}_{4}$ was added to the media to final concentration of $10 \mu \mathrm{M}$ because all insect EcRs and USPs are under CUP1 promoter. One hundred microliters of the cell culture were spiked to each well of a 96-well microtiter plate. To each well, $2 \mu$ l ligand solution (diluted in DMSO) were added. For control wells, $2 \mu \mathrm{l}$ DMSO were also added. The final concentration of the tested compounds in the media was $10 \mu \mathrm{M}$. Cells were incubated in the presence of a ligand in a shaker at $30^{\circ} \mathrm{C}$. After $4 \mathrm{~h}$ of incubation, $100 \mu \mathrm{l}$ of $2 \times$ ' $\mathrm{Z}$ ' Sarcosine-O-nitrophenyl- $\beta$-Dgalactopyranoside (ONPG) buffer $(120 \mathrm{mM}$ $\mathrm{Na}_{2} \mathrm{HPO}_{4}, 80 \mathrm{mM} \mathrm{NaH} \mathrm{PO}_{4}, 20 \mathrm{mM} \mathrm{KCl}, 2 \mathrm{mM}$ $\mathrm{MgSO}_{4}, 100 \mathrm{mM} \beta$-mercaptoethanol, pH 7.0, $0 \cdot 4 \%$ lauroyl sarcosine, $4 \mathrm{mg} / \mathrm{ml}$ ONPG) were added to each well and the plate was further incubated at $37^{\circ} \mathrm{C}$. The $2 \times$ ' $Z$ ' Sarcosine-ONPG buffer was freshly prepared or stored at $-20{ }^{\circ} \mathrm{C}$ before use. After incubation at $37^{\circ} \mathrm{C}$ for $1 \mathrm{~h}\left(\mathrm{t}_{\text {incubation }}\right)$, the reaction was stopped by the addition of $100 \mu \mathrm{l}$ quenching solution $0.5 \mathrm{M} \mathrm{Na} \mathrm{Na}_{2} \mathrm{CO}_{3}$ and $\mathrm{OD}_{405}$ $\left(\mathrm{OD}_{\text {reaction }}\right)$ and $\beta$-galactosidase activity was measured in microplate reader (Biotek). As in all assays, the $\mathrm{OD}$ of the cultures was standardized as $0 \cdot 1$ and time for incubation with the $\beta$-galactosidase substrate ONPG was $1 \mathrm{~h}$. The values presented in Figs 2-7 are equal to the median of optical density $\mathrm{OD}_{405}$ of at least eight independent reactions, multiplied by 1000 .

\section{RESULTS}

\section{Ligand-independent transactivation of AaEcR}

Previous analyses using the in vitro insect cell line or in vitro-transcribed-translated receptors have shown that the formation of the EcR-USP heterodimer is required for both the DNA and ligand-binding activities (Yao et al. 1993, Kothapalli et al. 1995, Kapitskaya et al. 1996). In contrast, expression of Drosophila EcR alone in yeast has been shown to activate constitutive transcription of a reporter gene containing EcREs in its 5'-regulatory region (Dela Cruz \& Mak 1997). In our experiments, we observed that expression of the full-length AaEcR alone induced transcription of the ecdysone reporter gene, irrespective of the absence or the presence of ligands (Fig. 2). This strong constitutive transactivation by the AaEcR was observed in the absence of USP (Fig. 2). The constitutive transcription activity of $\mathrm{AaEcR}$ was specific for a reporter gene harboring the EcRE. When AaEcR was co-expressed with a reporter plasmid YRpE2 (Lyttle et al. 1992) containing a reporter gene that was under regulation of the estrogen response element, no induced transcription was observed (data not shown). Even in the absence of copper induction, when expression 


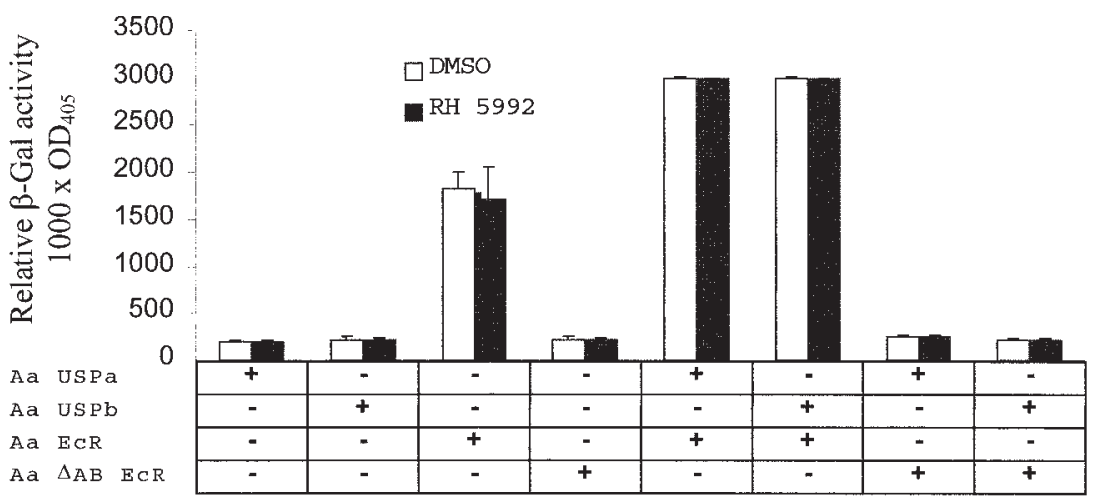

FIGURE 2. Transcriptional activation of A. aegypti (Aa) USP and EcR. The yeast strains carrying the reporter plasmid that contains six EcREs coupled with $E$. coli $\beta$-galactosidase ( $\beta$-Gal) gene in the absence or the presence of different combinations of plasmids for expression of AaUSP a or b isoforms or $\mathrm{Aa} \Delta \mathrm{USP}$ and EcR receptor. The relative transactivation activity in the absence or the presence of $10 \mu \mathrm{M}$ RH-5992 was measured as described in Materials and Methods. The data are presented as a median of at least eight independent experiments, plus its standard deviation.

of $\mathrm{AaEcR}$ barely was observed in western blot, the constitutive transcription activity effect of AaEcR on the EcRE reporter gene was still observed (data not shown). Co-expression of $\mathrm{AaEcR}$ with either $\mathrm{AaUSPa}$ or AaUSPb resulted in stronger constitutive transactivation than was achieved by AaEcR alone (Fig. 2). However, the expression of either AaUSP isoform alone did not induce the reporter gene, clearly indicating that the presence of the full length of AaEcR was required for constitutive transactivation (Fig. 2). Surprisingly, removal of the A/B domain from AaEcR resulted in complete eradication of this constitutive transactivation activity. The constitutive transactivation ability of the truncated AaEcR was not restored by coexpression either with AaUSP or with mammalian RXRs (Figs 2 and 3). Thus the A/B domain of AaEcR presumably has a role as a ligandindependent transcription activator, at least in yeast.

Previously, Perera and coworkers (1999) showed that removal of the A/B domain of the $C$. fumiferana EcR does not affect either DNA or ligand binding activity. This suggests that the lack of the transactivation activity of Aa $\Delta \mathrm{EcR}-\mathrm{USP}$ could be due to the deficiency in communication between the heterodimer bound to EcRE and the yeast general transcription machinery. One of the possible reasons would be the lack of proper co-factors, which mediate such communication. Therefore we tested co-factors that could activate the liganddependent response of the Aa $\triangle \mathrm{EcR}-\mathrm{USP}$ or Aa $\Delta E c R-R X R$ heterodimers in yeast.

\section{Reconstruction of ligand-dependent AaEcR-USP and AaEcR-RXR transcription in yeast}

We speculated that $\mathrm{Aa} \Delta \mathrm{EcR}-\mathrm{USP}$ and $\mathrm{Aa} \Delta \mathrm{EcR}-$ RXR could form a heterodimer with the ability to bind to the ligand but not initiate transcription. One possible reason for this inability to initiate transcription is the lack of co-factor(s) that serve as a 'bridge' between the heterodimer and the yeast general transcription complex binding to the TATA box of the CYC1 promoter. It has been shown previously that RXRs can interact with GRIP1, forming a protein link between ligand-activated nuclear receptors bound to cognate hormone response elements and the transcription initiation apparatus (Walfish et al. 1997). Therefore, the role of GRIP1 in the liganddependent transactivation assay for EcR-USP and EcR-RXR was tested. Transactivation of $\mathrm{Aa} \Delta \mathrm{EcR}-\mathrm{USP}$ and $\mathrm{Aa} \Delta \mathrm{EcR}-\mathrm{RXR}$ in the presence GRIP1 was observed in response to RH-5992 (Figs $3 \mathrm{~A}$ and $\mathrm{B})$. This suggests that GRIP1 can interact with both $\mathrm{Aa} \Delta \mathrm{EcR}-\mathrm{USP}$ and $\mathrm{Aa} \Delta \mathrm{EcR}-\mathrm{RXR}$ complexes. GRIP1 obviously acts differently than the $\mathrm{A} / \mathrm{B}$ domain of EcR, because the full-length AaEcR initiates transcription in the absence of EcR ligands. We have noted that, in order to achieve liganddependent transactivation activity, it requires the presence of all three components: GRIP1, EcR and RXR or its counterpart, USP; without one of them, transactivation activity was not observed (Fig. 3). 


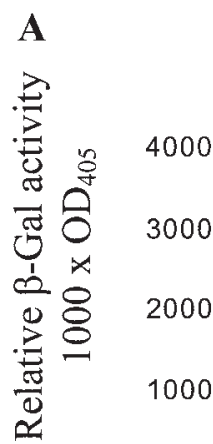

$\square$ DMSO

RH 5992

\begin{tabular}{|c|c|c|c|c|c|c|c|c|c|c|}
\hline Aa $\triangle A B E C R$ & + & $=$ & - & - & + & + & + & + & + & + \\
\hline$R \times R \alpha$ & - & + & - & - & + & - & - & + & - & - \\
\hline$R \times R \beta$ & - & - & $t$ & $=$ & - & + & - & - & + & - \\
\hline $\mathrm{R} \times \mathrm{R} \gamma$ & - & - & - & + & - & - & + & - & - & + \\
\hline GRIP 1 & + & + & + & $t$ & - & - & - & + & + & + \\
\hline
\end{tabular}

\section{B}

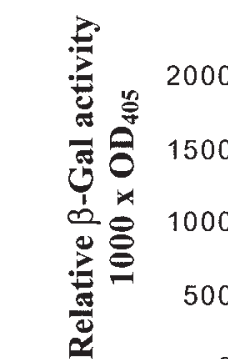

Aa $\triangle A B E C R$

Aa USPa

Aa USPb

Aa $\triangle A B \cup S P$

GRIP 1

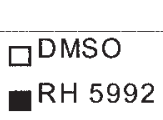

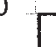

\begin{tabular}{|c|c|c|c|c|c|c|c|c|}
\hline+ & + & + & - & - & - & + & + & + \\
\hline+ & - & - & + & - & - & + & - & - \\
\hline- & + & - & - & + & - & $=$ & + & - \\
\hline- & - & + & - & - & + & - & - & + \\
\hline - & $=$ & - & + & + & + & + & 7 & + \\
\hline
\end{tabular}

FIGURE 3. Transactivation of A. aegypti (Aa) EcR with A/B domain deletion in combination with mammalian RXRs or with AaUSPs in the absence and the presence of GRIP1. (A) Transactivation of Aa $\Delta$ USP in combination with RXRs. (B) Transactivation of $\mathrm{Aa} \triangle \mathrm{EcR}$ in combination with different variants of mosquito USP. The yeast strains carrying the reporter plasmid that contains six EcREs coupled with $E$. coli $\beta$-galactosidase $(\beta$-Gal) gene in the absence or the presence of different combinations of plasmids for expression of Aa $\Delta \mathrm{EcR}$, RXRs or AaUSP receptors and GRIP1. The final concentration of RH-5992 was $10 \mu \mathrm{M}$. $\beta$-Galactosidase assays were performed as described in Materials and Methods. The data are presented as a median of at least eight independent experiments, plus its standard deviation.

\section{Transactivation response to different ecdysteroid analogs}

The ligand-dependent transactivation system in yeast for insect EcRs is derived from the following components: ecdysone response elements (DNA sequences to which EcR-USP binds), EcR itself, the partners of EcR (USP or RXR), co-activators, and the EcR ligands. A change in one in these components will affect the transactivation response. We investigated the effect of different ecdysteroid analogs in combination with the different RXRs and USPs, while keeping EcR, co-activator and the response element constant. The ecdysteroid analogs examined were different chemicals derived from the RH-5849 that has been thoroughly studied (Wing et al. 1988, Dhadialla et al. 1998). The derivatives of RH-5849 have a spectrum of activities relative to the specific insect EcR. We tested six proprietary compounds from the Rohm \& Haas Company (coded as RH-1, RH-2, RH-3 RH-4 RH-5 and RH-6), four other compounds that have been published elsewhere (RH-0345, 

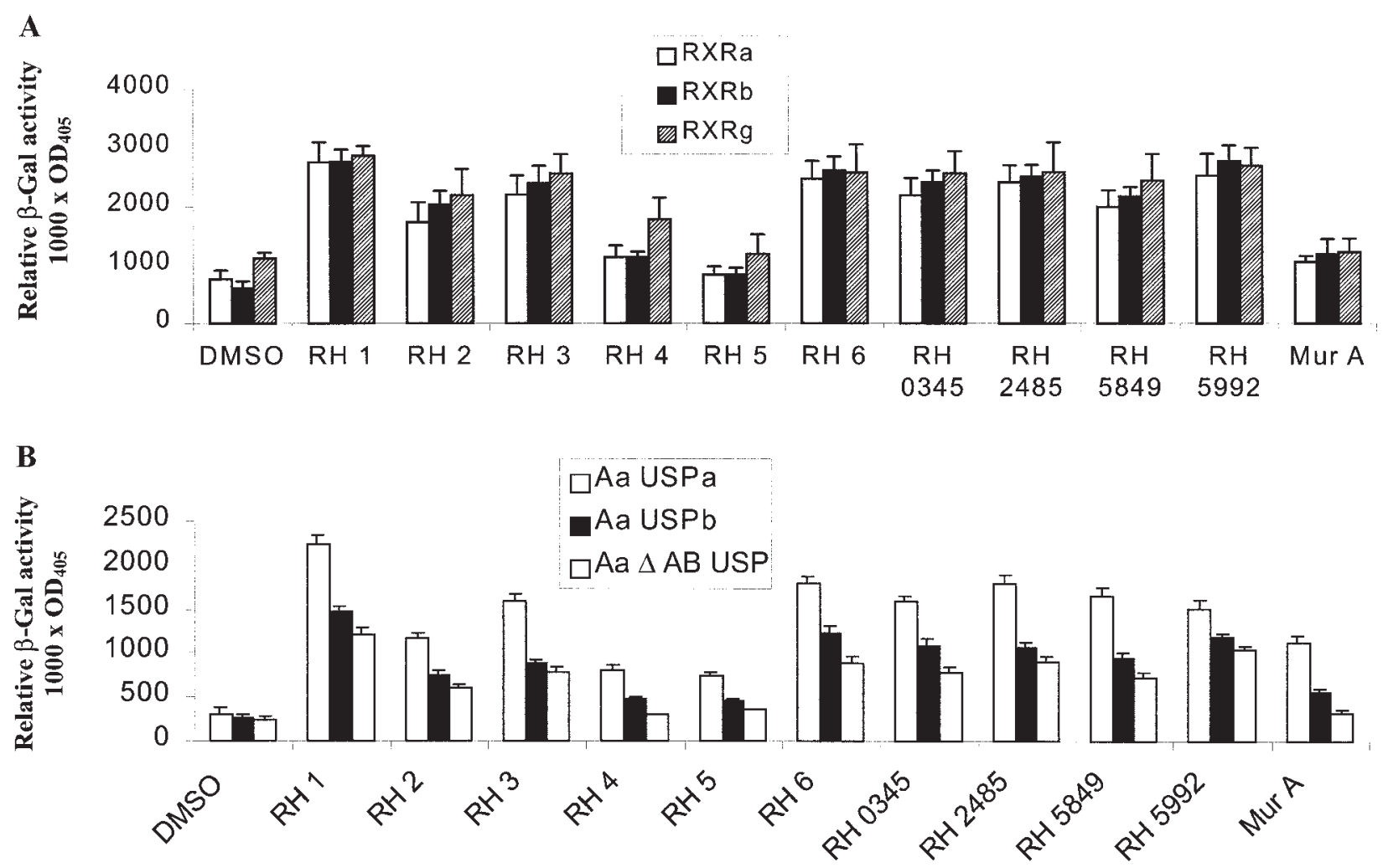

FIGURE 4. Transcriptional activation induced by different ecdysteroid agonists. (A) Transactivation activity of $\mathrm{Aa} \Delta \mathrm{EcR}-\mathrm{RXR}(\alpha, \beta$ or $\gamma)$. (B) Transactivation activity of $\mathrm{Aa} \Delta \mathrm{EcR}-\mathrm{USP}$ (a, b or $\Delta \mathrm{A} / \mathrm{B}$ variants). The yeast strains carrying the reporter plasmid that contains six EcREs coupled with $E$. coli $\beta$-galactosidase $(\beta$-Gal) gene in combination with the presence of different plasmids for expression of RXRs $(\alpha, \beta$ or $\gamma$ ) or USPs (AaUSPa, AaUSPb or $\mathrm{Aa} \Delta \mathrm{USP}$ ) and $\mathrm{Aa} \Delta \mathrm{EcR}$ and GRIP1. The final concentration of all ecdysteroid analogues was $10 \mu \mathrm{M}$.

$\beta$-Galactosidase assays were performed as described in Materials and Methods. The data are presented as a median of at least eight independent experiments, plus its standard deviation.

RH-2485, RH-5849 and RH-5992; Dhadialla et al. 1998), and muristerone A (Mur A).

As shown in Fig. 3A, the RXR-Aa $\triangle$ EcR-GRIP1 complex induced a high basal transcription background; however, the presence of ecdysteroid compounds resulted in further increased transcription of the reporter gene. Different RXRs have different effects on transcription. The presence of the $\operatorname{RXR} \gamma$ results in the highest background. As shown in Fig. 4A, different EcR-RXRs responded differently to the test compounds. The weakest compounds are RH-4, RH-5 and Mur A.

Compared with the presence of RXR, that of AaUSP as a heterodimer partner of the AaEcR did not result in a high basal transcription background (Figs $3 \mathrm{~B}$ and $4 \mathrm{~B}$ ). However, the heterodimers of $\mathrm{Aa} \Delta \mathrm{EcR}$ with different USPs (USPa, USPb, and $\Delta \mathrm{USP}$ ) resulted in varying responses to the

www.endocrinology.org ecdysteroid compounds. The strongest response was observed for USPa and the weakest for $\triangle \mathrm{USP}$ (Figs $3 \mathrm{~B}$ and $4 \mathrm{~B}$ ). The only difference between the $\mathrm{USPa}$ and $\mathrm{USPb}$ is in the N-terminal: the USPa is longer than $\mathrm{USPb}$ and there is some difference in the amino acid sequence of the $\mathrm{N}$-terminal (Kapitskaya et al. 1996). The differences in the response of $\mathrm{Aa} \Delta \mathrm{EcR}-\mathrm{USPa}$ and $\mathrm{Aa} \Delta \mathrm{EcR}-\mathrm{USPb}$ (Fig. 4B) suggest that the A/B domain of the USP can affect the ligand-dependent response of the entire EcR-USP heterodimer. Although EcR receptor partners (USP or RXR) can enhance or reduce the response of the heterodimers to the ligands, overall we have observed consistent potency for different compounds using various RXRs or USPs as EcR partners (Fig. 5). For all EcR-RXRs or EcR-USPs, the compounds RH-4 and RH-5, in addition to Mur A, gave the weakest responses (Fig. 4). 


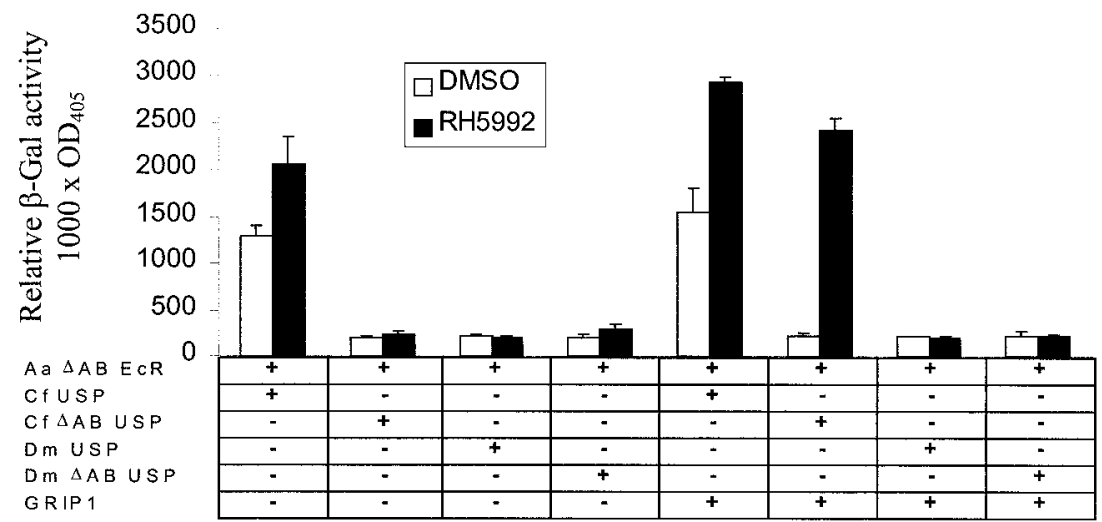

FIGURE 5. Transactivation of mosquito $\mathrm{Aa} \Delta \mathrm{EcR}$ in combination with different USP variants from spruce budworm and fruit fly in the absence and the presence of co-activator GRIP1. The yeast strains carrying the reporter plasmid that contains six EcREs coupled with $E$. coli $\beta$-galactosidase $(\beta$-Gal) gene in combination with the presence of different of plasmids for expression of USPs, $\mathrm{Aa} \Delta \mathrm{EcR}$ and GRIP1. The final concentration of RH-5992 was $10 \mu \mathrm{M}$. $\beta$-Galactosidase assays were performed as described in Materials and Methods. The data are presented as a median of at least eight independent experiments, plus its standard deviation.

\section{Effect of different USPs in ligand-dependent transactivation of mosquito EcR}

Although RH-5992 is a ligand for EcR, the receptor is able to bind to the ligand only in the context of a heterodimer with USP or RXR. As we have shown in Fig. 3, different RXRs have distinctive activities in combination with $\mathrm{Aa} \Delta \mathrm{EcR}$. Three variants of mosquito USP also give different levels of response in combination with $\mathrm{Aa} \Delta \mathrm{EcR}$. When EcR agonists were tested in the context of USPs, these variants gave similar responses; the differences were only in level of induction. The best result was observed for USPa (Figs 3 and 4B). As these variants differ from each other only in the $\mathrm{A} / \mathrm{B}$ domain $(\mathrm{AF}-1)$, the differences in level of ligand induction suggested that the AF-1 domain may be working in concert with $\mathrm{AF}-2$ in response to ligands. We further investigated how AaEcR responds to ecdysteroid ligands in combination with different USPs derived from spruce budworm $C$. fumiferana and fruit fly D. melanogaster.

As shown in Fig. 5, the presence of the full-length $C$. fumiferana USP, but not D. melanogaster USP, in combination with $\mathrm{Aa} \Delta \mathrm{EcR}$ resulted in constitutive transcription of the reporter gene. One must note that the expression of the $\mathrm{Aa} \Delta \mathrm{EcR}$ or CfUSP alone did not result in either constitutive or ligand-dependent transactivation. Surprisingly, deletion of the A/B domain of the CfUSP resulted in elimination of observed constitutive transactivation, suggesting that the AF-1 domain of the CfUSP modulates constitutive transcription of the reporter gene, but only in combination with EcR. In Fig. 5, we also showed that the presence of GRIP1 co-activators was absolutely required for the ligand-dependent response of $\mathrm{Aa} \Delta \mathrm{EcR}-\mathrm{Cf} \Delta \mathrm{USP}$. In contrast to CfUSP expression, that of DmUSP in combination with $\mathrm{Aa} \triangle \mathrm{EcR}$ did not result in transcription induction. Even in the presence of GRIP1 the heterodimer complexes Aa $\Delta$ EcRDmUSP and Aa $\Delta \mathrm{EcR}-\mathrm{Dm} \Delta \mathrm{USP}$ failed to give a ligand-dependent response. We also observed that the heterodimer of $\mathrm{Aa} \Delta \mathrm{EcR}$ with $\mathrm{Cf} \Delta \mathrm{USP}$ gave a stronger ligand-dependent response than the heterodimers of $\mathrm{Aa} \Delta \mathrm{EcR}$ with its own USP partners (Figs 3 and 5). These data suggest that USP can modulate the transactivation activity of the EcR and affect the EcR response to its ligands.

\section{Role of other co-activators/co-repressors in the ligand-dependent response of insect EcR-USP complexes}

A series of co-activators and co-repressors have been cloned from mammals and from $D$. melanogaster. They include: Drosophila CBP (Akimaru et al. 1997), mouse CBP (Chrivia et al. 1993), GRIP1 (Hong et al. 1996), SRC1A (Onate et al. 1995), RIP140 (Cavailles et al. 1995), SMRT (Chen et al. 1996), and N-coR (Horlein et al. 1995). Some of these enhance the ligand-dependent transactivation response in yeast-based systems (Joyeux et al. 1997, 
A

2000

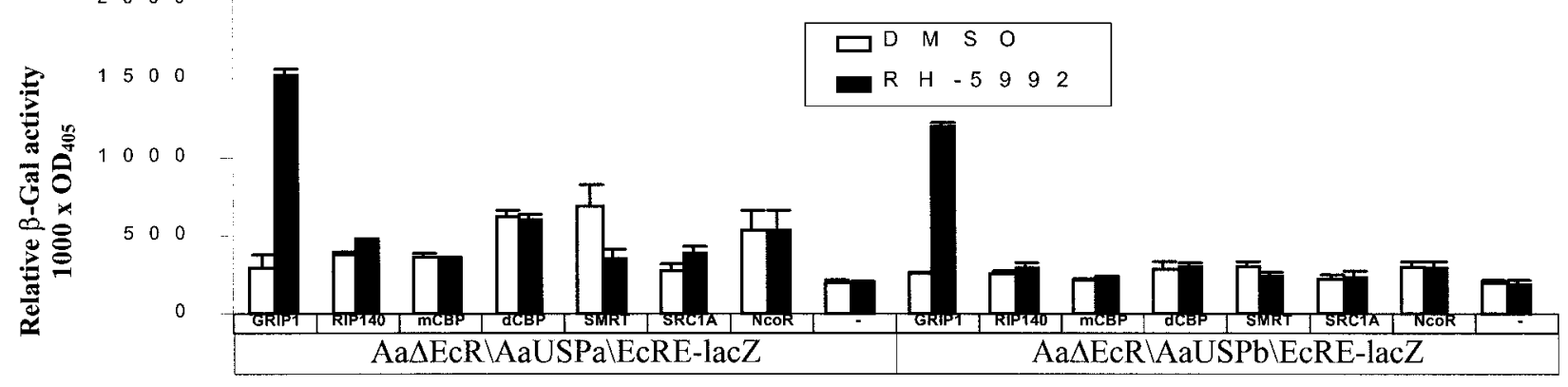

B

3500

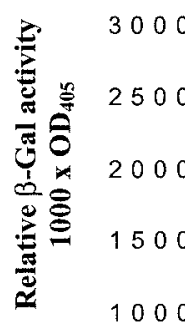

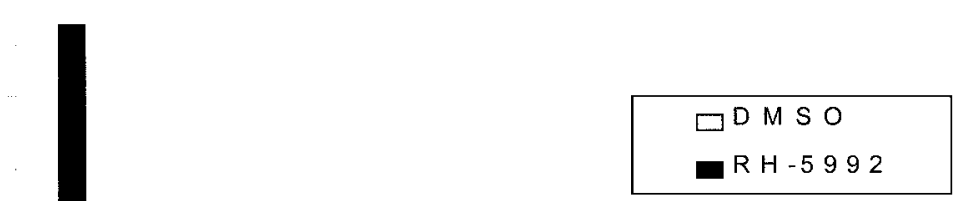

1000
500

0

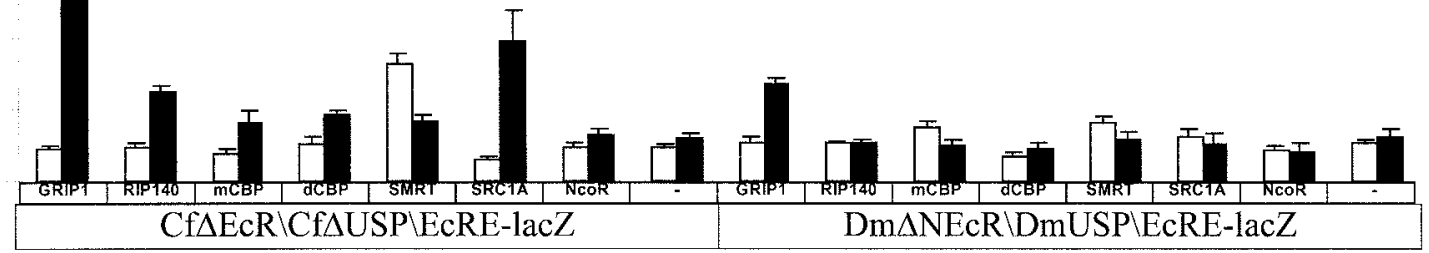

FIGURE 6. Roles of co-activators and co-repressors in transactivation of Aa $\Delta \mathrm{EcR}$. (A) Interaction of different transcriptional factors in combination with the heterodimers $A a \Delta E c R-U S P a$ or $A a \Delta E c R-U S P b$. (B) Interaction of different transcriptional factors in combination with the heterodimers Cf $\Delta$ EcR-Cf $\Delta$ USP or Dm $\Delta$ NEcR-DmUSP. The yeast strains carrying the reporter plasmid that contains six EcREs coupled with E. coli $\beta$-galactosidase ( $\beta$-Gal) gene in combination with the presence of different of plasmids for expression of insect EcR and USPs in combination with co-activators/co-repressors. The final concentration of RH 5992 compound was $10 \mu \mathrm{M}$. $\beta$-Galactosidase assays were performed as described in Materials and Methods. The data are presented as a median of at least eight independent experiments, plus its standard deviation.

Walfish et al. 1997). Co-repressor-mediated liganddependent repression of nuclear receptors has not been observed in yeast to date. It is useful to know whether these co-activators/co-repressors are able to influence the activity of $\mathrm{Aa} \Delta \mathrm{EcR}-\mathrm{USPa}$ and $\mathrm{Aa} \triangle \mathrm{EcR}-\mathrm{USPb}$ in the presence and absence of ligands in yeast. Recently, we have reported a construction of ligand-dependent transactivation for spruce budworm CfEcR-USP in the presence of GRIP1 in yeast (Tran et al. 2001). In this study we tested the effect of other co-activators/co-repressors on CfEcR-USP. Although SRC1A belongs to the family of co-activator GRIP1, we did not observe activity similar to that observed for GRIP1 for mosquito EcR-USP (Fig. 6A). In contrast, coactivator SRC1A expressed was able to induce a ligand-dependent transactivation of $\mathrm{Cf} \Delta \mathrm{EcR}-$
$\mathrm{Cf} \Delta \mathrm{USP}$ in yeast (Fig. 6B). (SRC1A expressed in the same plasmid was also able to enhance transactivation of the human thyroid hormone receptor, hTR $\beta$, in response to tri-iodothyronine (P G Walfish, unpublished data).) Similar to mosquito $\mathrm{Aa} \Delta \mathrm{EcR}-\mathrm{USP}$, only GRIP1, but not SRC1A, gave an effect for D. melanogaster Dm $\Delta \mathrm{NEcR}-\mathrm{USP}$ (Fig. $6 \mathrm{~B})$. The small effect of RIP140 and CBP was observed when combined with $\mathrm{Cf} \Delta \mathrm{EcR}-\mathrm{Cf} \Delta \mathrm{USP}$, but not with mosquito and fruit fly EcR-USP complexes (Fig. 6). (In other yeast transactivation systems, RIP140 was able to enhance the liganddependent response of ER (Sheeler et al. 2000).) This suggests that the co-activators GRIP1, SRC1A, RIP140 and CBP can distinctively interact with insect EcR-USP complexes in yeast. For the co-repressor $\mathrm{N}$-coR, no effect was observed in 


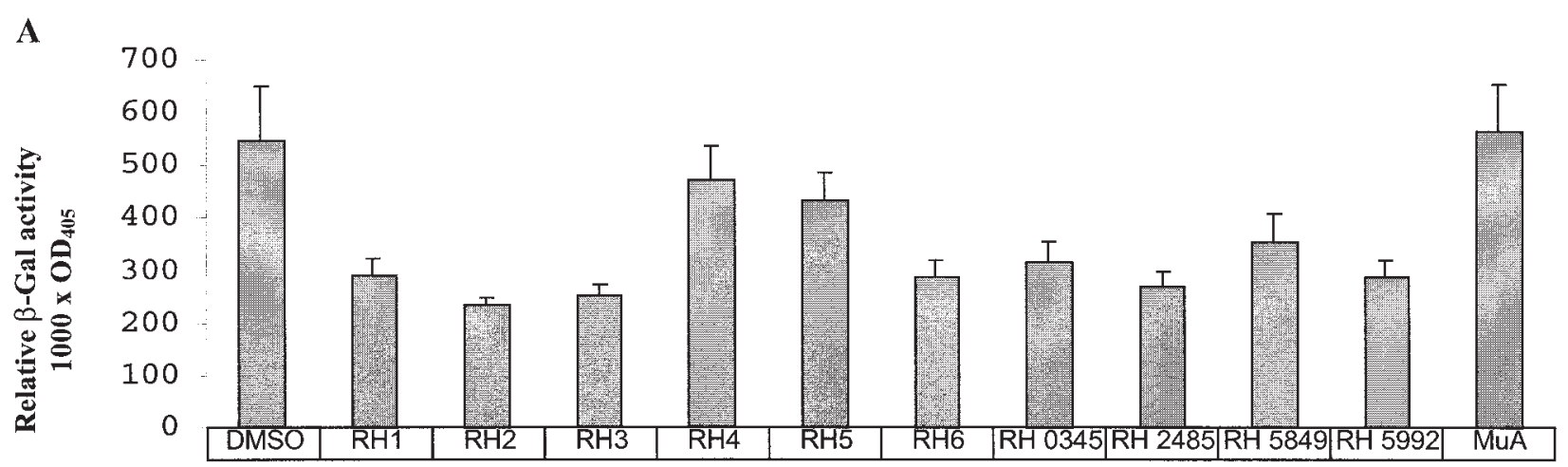

B

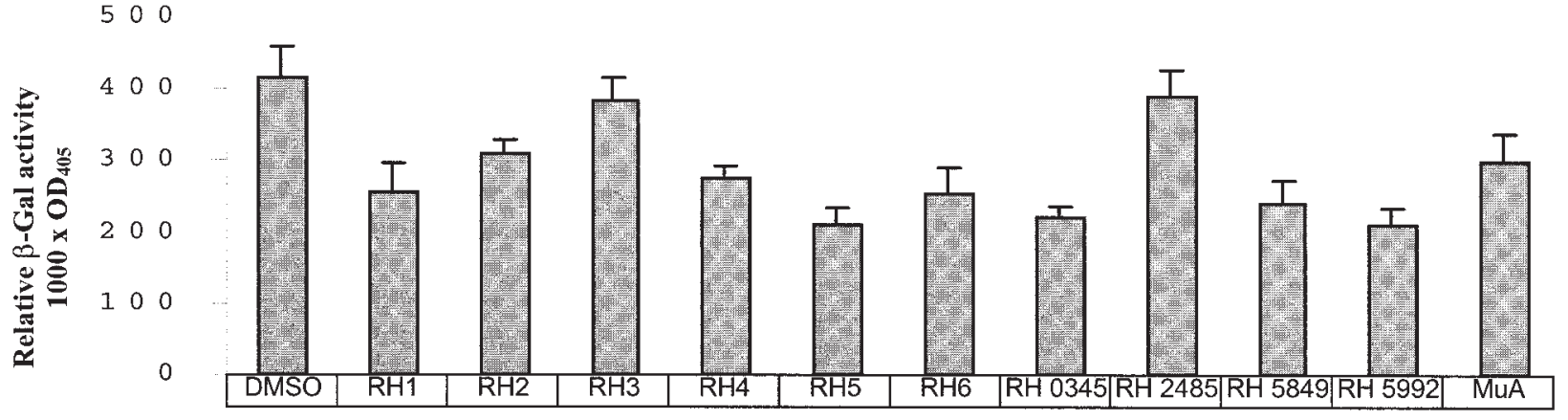

FIGURE 7. Roles of co-repressor SMRT in transactivation of insect EcR and USP receptors. (A) Impact of different ecdysteroidal analogs on transcription reduction of the Aa $\triangle E c R-U S P a-S M R T$ complex. (B) Impact of different ecdysteroidal analogs on transcription reduction of the $\mathrm{Cf} \Delta \mathrm{EcR}-\mathrm{Cf} \Delta \mathrm{USP}-\mathrm{SMRT}$ complex. The yeast strains carrying the reporter plasmid that contains six EcREs coupled with E. coli $\beta$-galactosidase ( $\beta$-Gal) gene in combination with the presence of different of plasmids for expression of insect USP and EcR and SMRT. The final concentration of all ecdysteroid analogues was $10 \mu \mathrm{M}$. $\beta$-Galactosidase assays were performed as described in Materials and Methods. The data are presented as a median of at least eight independent experiments, plus its standard deviation.

either EcR-USP combination (Fig. 6). However, for the $A a \Delta \mathrm{EcR}-\mathrm{USPa}$ and $\mathrm{Cf} \Delta \mathrm{EcR}-\Delta \mathrm{USP}$, the presence of co-repressor SMRT increased the level of transcription of the reporter gene (Fig. 6). When the ligand RH-5992 was added, the transcription of the reporter gene was reduced up to twofold (Fig. 6). We tested whether different ecdysteroid analogs can modulate the SMRT effect. As shown in Fig. 7, the more potent compounds are those with greater levels of ligand-dependent suppression. The effect is quite opposite to that observed in the presence of co-activator GRIP1 (Fig. 4). We have also noted that potencies of 11 test compounds for $\mathrm{Cf} \Delta \mathrm{EcR}-$ $\triangle$ USP-GRIP1 and Aa $\Delta$ EcR-USPa-GRIP1 were different (Table 1); for the Cf $\Delta \mathrm{EcR}-\Delta \mathrm{USP}-\mathrm{SMRT}$ and $\mathrm{Aa} \Delta \mathrm{EcR}-\mathrm{USPa}-\mathrm{SMR} T$ complexes these compounds also have different effects on transactivation reduction (Fig. 7). As shown in Fig. 2, AaEcR-USP induces constitutive transcription of the reporter gene. We tested whether SMRT is able to suppress the transactivation activity of the heterodimer. SMRT and AaEcR-USP were co-expressed in yeast in the presence of reporter gene. In the presence and absence of the ligand RH-5992, the constitutive transcription was not suppressed by SMRT (data not shown). Thus the liganddependent repression effect of SMRT was observed only in the context of Aa $\Delta \mathrm{EcR}-\mathrm{USP}$, and not for AaEcR-USP.

\section{DISCUSSION}

\section{Ligand-independent transactivation and the role of $\mathrm{AF}-1$ in the ligand-dependent response}

Overall, alignment of different insect EcRs and USPs revealed a high homology in their DNA binding domains. However, other regions such as $\mathrm{A} / \mathrm{B}(\mathrm{AF}-1)$ and $\mathrm{D} / \mathrm{E} / \mathrm{F}(\mathrm{AF}-2)$ shared homology to a lesser extent, suggesting that these regions 
TABLE 1. Comparison between data of transactivation activity in insect cells L57 and in yeast systems (fold induction)

Fold induction

\begin{tabular}{|c|c|c|c|}
\hline & \multirow{2}{*}{$\frac{\text { Insect cells }}{\text { CfEcR-DmUSP* }}$} & \multicolumn{2}{|l|}{ Yeast systems } \\
\hline & & $\mathrm{Cf} \Delta \mathrm{EcR}-\mathrm{Cf} \Delta \mathrm{USP}^{*}$ & $\mathrm{Aa} \Delta \mathrm{EcR}-\mathrm{AaUSPa}$ \\
\hline \multicolumn{4}{|c|}{ Compounds } \\
\hline DMSO & $1 \cdot 0$ & $1 \cdot 0$ & $1 \cdot 0$ \\
\hline Mur A & 141 & $1 \cdot 9$ & $3 \cdot 9$ \\
\hline RH-1 & $54 \cdot 0$ & $6 \cdot 5$ & $7 \cdot 6$ \\
\hline RH-2 & $47 \cdot 0$ & $4 \cdot 2$ & $3 \cdot 9$ \\
\hline RH-3 & $30 \cdot 0$ & $5 \cdot 1$ & $5 \cdot 3$ \\
\hline RH-4 & $0 \cdot 9$ & $1 \cdot 0$ & $2 \cdot 7$ \\
\hline RH-5 & $21 \cdot 0$ & $6 \cdot 7$ & $2 \cdot 5$ \\
\hline RH-6 & $16 \cdot 5$ & $5 \cdot 2$ & $6 \cdot 1$ \\
\hline RH0345 & $23 \cdot 6$ & $5 \cdot 0$ & $5 \cdot 3$ \\
\hline RH2485 & $51 \cdot 0$ & $5 \cdot 4$ & $6 \cdot 1$ \\
\hline RH5849 & $27 \cdot 7$ & $3 \cdot 4$ & $5 \cdot 6$ \\
\hline RH5992 & $43 \cdot 6$ & $6 \cdot 3$ & $5 \cdot 1$ \\
\hline
\end{tabular}

underwent significant divergence during evolution (Fig. 1). The divergence in the AF-1 and AF-2 domains of EcR and USP may modulate the EcR-USP complex in response to ecdysteroids. The evolutionary divergence in these domains in different insects can be exploited in designing ligands that may act in a species-specific manner.

Ecdysteroid-dependent induction of transcription in insects is not dependent solely on the EcR-USP complex, but also on a set of EcR-USP-interacting co-activators/co-repressors that might be expressed specifically in each tissue. In contrast to insect cells, yeast does not contain any nuclear receptors or homologues of co-activators/co-repressors. When EcR is expressed in yeast, the components of co-activators/co-repressors are absent. Also in contrast to what happens in insects, we have observed that expression of $\mathrm{AaEcR}$ alone in yeast, similar to what has been observed for DmEcR (Dela Cruz \& Mak 1997), induces ligand-independent transactivation. Thus expression of $\mathrm{DmEcR}$ and $\mathrm{AaEcR}$ in yeast resulted in ligand-independent transcription of the EcRE reporter gene. However, expression of another EcR from spruce budworm $(\mathrm{CfEcR})$ in the same expression yeast vector did not induce transcription of the reporter gene (Tran et al. 2001). Interestingly, both the fruit fly (D. melanogaster) and the yellow fever mosquito

www.endocrinology.org
(A. aegypti) belong to the class Diptera, whereas spruce budworm is a lepidopteran. Co-expression of $\mathrm{AaEcR}$ and AaUSP further enhances the induction of transcription of the reporter gene (Fig. 2). These observations suggest that there exist co-repressors in insects that suppress constitutive transactivation activity of EcR. One of the possible candidates as a co-repressor is insect SMRTER, which has been identified as an EcR-interacting protein and a homolog of the mammalian co-respressors SMRT and N-coR (Tsai et al. 1999). Surprisingly, deletion of the $\mathrm{A} / \mathrm{B}$ domain of $\mathrm{EcR}$ eliminates the constitutive transcription activity (Fig. 2). The ligand-dependent transactivation was observed only when a new co-activator (GRIP1) was added to the EcR-USP complex (Fig. 3). In the light of these observations, in the absence of ligand the transactivation activity of the A/B domain (AF-1) in EcR in insects may be masked by co-repressors, whereas this activity is not present in yeast cells because of a lack of co-repressor.

The role of the AF-1 domain in ligandindependent transactivation is well documented. For example, the AF-1 domain of $\mathrm{ER}$ can be activated by an EGF-triggered phosphorylation pathway (El-Tanani \& Green 1997). The activity of the AF-1 domain is modulated by interaction with co-activators or co-repressors (Wilkinson \& Towle 
1997, Onate et al. 1998). We have also demonstrated that the full length of CfUSP can also induce ligand-independent transactivation. However, in contrast to $\mathrm{AaEcR}$, the constitutive transactivation activity of CfUSP is dependent on the presence of EcR (Fig. 5). Deletion of the A/B (AF-1) domain of the CfUSP results in the elimination of constitutive transactivation. These observations suggest that the AF-1 domains of the AaEcR or CfUSP possess transactivation functions. Recently, we also demonstrated that deletion of the AF-1 domain of the $\mathrm{CfEcR}$ enhanced ligand-dependent transactivation in yeast, suggesting an inhibitory activity of the AF-1 domain of the CfEcR (Tran et al. 2001). As we have demonstrated above, AaEcR alone can induce transcription of the reporter gene harboring EcREs in the promoter (Fig. 2). The effect of the $\mathrm{AaEcR}$ is specific for the reporter with EcRE elements as expression of the AaEcR did not induce transcription of the reporter gene with estrogen response elements (data not shown). We can not exclude the possibility that yeast protein(s) exist that form heterodimers with $\mathrm{EcR}$ and bind to EcRE, even though a thorough search of the yeast protein database failed to find USP or RXR homologues. The functional $\mathrm{EcR}$ is commonly considered as a heterodimer consisting of two proteins, EcR and USP, yet our results clearly demonstrated that AaEcR alone, without USP, can function as a transactivator in yeast, in agreement with results obtained from DmEcR (Dela Cruz \& Mak 1997). In vitro transcribed DmEcR (Vogtli et al. 1998) and AaEcR (S F Wang and A S Raikhel, unpublished data) are able to bind DNA without USP. It is also conceivable that the EcR homodimer is able to communicate with yeast basal transcription machinery independently of the ligand.

The A/B domains of EcR and USP have roles not only in ligand-independent or constitutive transactivation, but also in the ligand-dependent response. In Fig. 3, we demonstrate that, although mosquito USPa, USPb and $\triangle \mathrm{USP}$ in combination with its $\Delta \mathrm{EcR}$ produce ligand-dependent transactivation, the efficacy or level of response was different for these variants. The best response to muristerone A was observed when $\mathrm{Aa} \Delta \mathrm{EcR}$ combined with AaUSPa. AaUSPa has an N-terminal sequence of MLKKEKPMLSVAAIIQAQGRWDRTLPLAGL, whereas $\mathrm{AaUSPb}$ has a sequence MDPSDR. The remainder of the amino acid sequences are identical, except for one amino acid change (Kapitskaya et al. 1996). In the yeast transactivation assay, the the magnitude of the responses to ecdysteroid analogs are in the order $\mathrm{Aa} \Delta \mathrm{EcR}-\mathrm{USPa}>\mathrm{Aa} \Delta \mathrm{EcR}-\mathrm{USPb}>\mathrm{Aa} \Delta \mathrm{EcR}-\mathrm{Aa} \Delta \mathrm{USP}$. There are at least two possible explanations for this.
Firstly, as the $\mathrm{A} / \mathrm{B}$ domain works in concert with the ligand-binding domain, deletion of the AF-1 domain or modification of the AF-1 domain can affect the potency of the ligands. Secondly, the AF-1 domain can interact with a co-activator (Ikonen et al. 1997, Wilkinson \& Towle 1997), resulting in a modification in the AF-1 domain that can result in an increase or decrease in transactivation. In any case, we have observed the impact of $\mathrm{N}$-terminal deletion on the activities of ligand binding and transactivation. In yeast, all three isoforms of $D$. melanogaster $\mathrm{EcR}$ showed ligandindependent transactivation of an ecdysone reporter gene, but the amount of activation was correlated with size of the $\mathrm{N}$-terminal $\mathrm{A} / \mathrm{B}$ domain present in the isoforms (Dela Cruz et al. 2000). Furthermore, we have observed ligand-independent transactivation of the $A a E c R$ and CfUSP (the effect of the latter being dependent on the presence of EcR). It is interesting to note that, when the $\mathrm{A} / \mathrm{B}$ domain of the AaEcR or CfUSP is fused, for example, with another CfEcR or DmUSP, the latter receptors do not exhibit ligand-independent transactivation. As we have noted, deletion of the $\mathrm{A} / \mathrm{B}$ domain of the AaEcR results in elimination of constitutive transactivation even in the presence of the AaUSPa or $\mathrm{AaUSPb}$; however, the presence of CfUSP leads to constitutive transactivation (Fig. 5). Recently, we have observed that CfUSP, when combined with $\mathrm{Dm} \Delta \mathrm{EcR}$ or $\mathrm{CfEcR}$ always induces constitutive transcription (Tran et al. 2001, and unpublished data). This raises the question of what happens if the A/B domain of the CfUSP is relocated to the $\mathrm{N}$-terminal of EcR.

\section{Response of EcR to its ligands is dependent on EcR partners}

USP is the insect counterpart of the mammalian $\mathrm{RXR}$. In vitro and in vivo data have shown that RXR could substitute for insect USP and form the heterodimer complex with EcR (Thomas et al. 1993, Yao et al. 1992, 1993, Suhr et al. 1998). However, in the absence of GRIP1 the heterodimer $\operatorname{RXR} \alpha(\beta$ or $\gamma)-\mathrm{Aa} \Delta \mathrm{EcR}$ did not exhibit transactivation activity in response to the ligand RH-5992 (Fig. 3). In combination with GRIP1 co-activator, all RXR-EcR heterodimers increased the basal transcription background and started to respond to the ligand RH-5992. In contrast to RXRs, insect USPs either provoke constitutive transcription (in the case of CfUSP) or do not increase background even in the presence of GRIP1 (Figs 3B, 4B and 5). In examining three variants of mosquito USPs, we have observed that USP with different N-termini can have different levels of response to ligands, in 
the following order: USPa $>\mathrm{USPb}>\Delta \mathrm{USP}$ (Fig. $3 \mathrm{~B})$. For both DmUSP variants in combination with mosquito $\mathrm{EcR}$, response to the ligand was not observed (Fig. 5). These results suggested that the A/B domain of USP could differentially modulate the transactivation function of EcR-USP. Thus the response of EcR to its ligands in transactivation is dependent not only on itself, but also on its partners. Modification in the AF-1 and divergence in the AF-2 of USP can affect the entire activity of the EcR-USP complex.

\section{Role of co-activators/co-repressors in receptor transactivation in yeast}

A series of nuclear receptors has been successfully reconstructed in yeast in the absence or the presence of co-activators such as RIP140, GRIP1 or SRC1 (Metzger et al. 1988, Mak et al. 1994, Butt \& Walfish 1996, Hong et al. 1996, Joyeux et al. 1997, Walfish et al. 1997). The co-activator SRC1 has been shown to co-ordinate interaction between $\mathrm{N}$ and $\mathrm{C}$-termini of the androgen receptor that are necessary for transcriptional activity (Ikonen et al. 1997). GRIP1 has been shown to interact in vitro in a ligand-dependent manner with thyroid receptor, retinoic acid receptor and RXR (Hong et al. 1997, Walfish et al. 1997, Anafi et al. 2000). In this work, we observed that the presence of GRIP1 is required for ligand-mediated transactivation function of EcR-USP or EcR-RXR in yeast (Figs 3, 4 and 5). As shown in Fig. 3, GRIP1 co-activator protein markedly increased the ability of EcRRXR heterodimers to transactivate the reporter genes harboring EcRE. For three subtypes of RXR receptors $(\alpha, \beta$ and $\gamma)$ in combination with $\mathrm{Aa} \Delta \mathrm{EcR}$ and GRIP, increased basal transcription of the reporter gene was observed (Fig. 3A), suggesting that the interaction between EcR-RXR and GRIP does not require the presence of a ligand. As a USP homolog to RXR, we demonstrated that not only does EcR-RXR interact in vivo with GRIP, but EcR-USP also can interact with GRIP1 (Fig. 3B). This is first example of mammalian co-activator working in concert with insect nuclear receptors. Previously, GRIP1 was shown to interact with TR-RXR or VDR-RXR heterodimers (Walfish et al. 1997). Our results demonstrated that GRIP could interact in vivo with both EcR-RXR and EcR-USP in a transactivation assay. Although SRC1A belongs to the family of co-activator GRIP1, we did not observe activity similar to that observed for GRIP1 for mosquito EcR-USP (Fig. 6A). However, for other EcR-USP complexes from spruce budworm $C$. fumiferana, an effect of co-activator SRC1A expressed in the same plasmid was observed (Fig. 6B), suggesting that coactivators GRIP1 and SRC1A can distinguish insect EcR-USP complexes in yeast.

The Drosophila homolog of co-repressor SMRT, SMRTER, has been cloned and shown to interact with EcR (Tsai et al. 1999). Here we have demonstrated that SMRT can genetically interact with the insect EcR-USP complex in yeast (Figs 6 and 7). Consequently, we plan to investigate the interaction of Drosophila SMRTER with EcR-USP using this transactivation system. The liganddependent function of SMRT in combination with the mosquito and spruce budworm EcR-USP heterodimers is presented in Figs 6 and 7. The $\mathrm{Aa} \triangle \mathrm{EcR}-\mathrm{AaUSPa}$ heterodimer alone did not induce transactivation of the reporter gene. The addition of SMRT to the heterodimer increased the basal transcription of the reporter gene. However, in the presence of ecdysteroidal analogs the acquired basal transcription was reduced (Figs $6 \mathrm{~A}$ and $7 \mathrm{~A}$ ). The more potent the ligand, the stronger the reduction that is observed. A similar effect was observed for $\mathrm{Cf} \Delta \mathrm{EcR}-\mathrm{Cf} \Delta \mathrm{USP}$. It has been proposed that co-repressors N-coR and SMRT repress transcription of targeted genes in the absence of ligands by interacting with nuclear receptors (Nagy et al. 1999). Upon the binding of nuclear receptors with ligands, co-repressors are released. In yeast twohybrid experiments, in the absence of an EcR ligand, both SMRT and N-coR have been shown to interact with C-terminal of D. melanogaster EcR (Thormeyer et al. 1999). The combination of SMRT with DmEcR yielded a much greater expression (more than 30-fold) of lac Z reporter gene compared with the combination of $\mathrm{N}$-coR with EcR (Thormeyer et al. 1999). This may explain why an effect of SMRT but not of N-coR was observed in our system (Fig. 6). Furthermore, Thormeyer et al. (1999) have shown that the presence of muristerone A leads to a decrease in the SMRT-EcR interaction in vitro. In another two-hybrid system mammalian assay, SMRT has been shown to interact with the heterodimeric DmEcR-USP complex (Tsai et al. 1999). Addition of hormone appears to disassociate SMRT completely from the heterodimer complex, therefore eliminating reporter activity (Tsai et al. 1999). Clearly, our data on SMRT in Figs 6 and 7, showing that addition of EcR ligand reduced the transactivation activity observed for EcR-USPSMRT complexes, are consistent with the published data of Tsai et al. (1999) and Thormeyer et al. (1999). Tsai and coworkers (1999) have also shown that the semi-lethal mutation A483T in the DmEcR eliminates 
EcR-USP interaction with SMRT. The alanine A483 residue is located within a highly conserved region of the LBD domain of Drosophila EcR (corresponding to A401 in A. aegypti EcR). Most probably, mutation A401T in the mosquito EcR will eliminate the SMRT effect that is shown in Fig. 7.

Nagy et al. (1999) reported the identity of complementary-acting signature motifs in SMRT and $\mathrm{N}-\mathrm{coR}$ that are sufficient for receptor binding and ligand-induced release. The motif contains a hydrophobic core (PhiPhixxPhi) similar to that found in nuclear receptor co-activators. Surprisingly, mutations in the amino acids that directly participate in co-activator binding disrupt the co-repressor association (Nagy et al. 1999). It would be interesting to test the effect of mutant variants of mammalian SMRT in the system, to determine whether the mutant SMRT still could induce basal transcription as presented in Fig. 6. Recently, an EcR-interacting protein, SMRTER, which is structurally divergent from but functionally similar to mammalian SMRT and N-coR, has been isolated (Tsai et al. 1999). SMRTER repression activity is mediated by interaction with $\operatorname{Sin} 3 \mathrm{~A}$, a repressor known to form a complex with the histone deacetylase Rpd3-HDAC. Mutant EcR that fails to interact with SMRTER results in a growth defect and lethality ('Tsai et al. 1999). It would be useful to know whether insect SMRTER acts similarly mammalian SMRT in interacting with EcR-USP as presented in Figs 6 and 7.

\section{Potential use of EcR transactivation assay in pesticide screening}

One of the purposes in developing a liganddependent transactivation system for $\mathrm{EcR}$ in yeast is to use it for identifying EcR ligands. Speciesspecific EcR ligand potentially can be used as a pesticide that targets insect specifically. Figure 4 demonstrated that different ligands have different abilities to induce AaEcR transactivation in yeast. The availability of several EcR transactivation systems will enable the rapid identification and comparison of ligands that are specific for each EcR. The availability of EcR-USP transactivation system for mosquito, spruce budworm and fruit fly EcRs (Figs 3 and 5) allowed us to compare the potencies of different compounds in different insect EcR. Table 1 presented the fold induction of transactivation in spruce budworm and mosquito EcRs by different compounds. As shown in the Table, the compound RH-4 is inactive for CfEcR both in yeast and in insect cell lines transfected with CfEcR receptor; however, some activity is observed for this compound when AaEcR is used. In contrast, RH-5 is a weak ligand for $\mathrm{AaEcR}$, but has a high potency for CfEcR transactivation. Furthermore, RH5849, the well-characterized ligand for insect EcR (Wing et al. 1988) has weak activity for CfEcR but strong activity for $\mathrm{AaEcR}$ transactivation. Among insect EcR and USP receptors, the ligand-binding domains are quite divergent, therefore it is possible that an EcR-targeting pesticide that is toxic to one type of insects is safe for others.

The yeast system reported here does not duplicate all aspects of the in vivo activity of EcR (e.g. the AF-1 region of EcR must be deleted), but it should prove to be a valuable tool for basic and applied studies. Further studies could involve the analysis of defined mutations analysis in which a specific amino acid of USP or EcR is changed, and investigation of how these mutations can affect responses to ligands and interactions with co-activators/co-repressors. The yeast system could be used to identify coactivators or co-repressors that act similarly to GRIP1 or SMRT respectively. We used the method for molecular profiling of several compounds possessing ecdysteroid-like properties. The results presented in Fig. 5 demonstrate that the method might serve as a system for screening of pesticides targeting EcR receptors. Searching for a USP ligand is of special interest. As we demonstrated, structural modification of USP or RXR affects the transactivation response to EcR ligand, therefore we believe that ligands of USP, if they exist, will also affect the transactivation response of EcR-USP to EcR ligand. Previously, Jones \& Sharp (1997) demonstrated that juvenile hormone III (two JH III structures, monoepoxide and bisepoxide) is able to bind to and change the conformation of USP. We examined the agonist and antagonist properties of the JH1, JH2 and $\mathrm{JH} 3$, in addition to phenoxycarb and methoprene, but did not see any agonist or antagonist activity of these proposed USP ligands. If firm conclusions are to be drawn on this subject, more intensive studies must be undertaken. We believe that the EcR-USP system could be used for studying ligands not only for EcR, but also for USP.

\section{ACKNOWLEDGEMENTS}

We would like to thank Reddy Palli, Bill Bergman and Shenfu Wang for helpful discussion and comments on the manuscript, and Jonathan Marsh for technical assistance. A Raikhel is supported by NIH Grant AI-36959 and P Walfish is supported by the Medical Research Council of Canada, Grant MT-14798. Thanks are also due to Mrs Adina Halter and Mrs Lindsay Tollen for their support in preparation of the manuscript. 


\section{REFERENCES}

Akimaru H, Chen Y Dai P, Hou DX, Nonaka M, Smolik SM, Armstrong S, Goodman RH \& Ishii S 1997 Drosophila CBP is a co-activator of cubitus interruptus in hedgehog signaling. Nature 386 735-738.

Allegretto EA, McClurg MR, Lazarchik SB, Clemm DL, Kerner SA, Elgort MG, Boehm MF, White SK, Pike JW \& Heyman RA 1993 Transactivation properties of retinoic acid and retinoid $\mathrm{X}$ receptors in mammalian cells and yeast. Correlation with hormone binding and effects of metabolism. Fournal of Biological Chemistry 268 26625-26633.

Anafi M, Yang Y-F, Berlev NA, Berger SL, Butt TR \& Walfish PG 2000 GCN5 and ADA adapter proteins regulate T3/GRIP coactivator dependent gene activation by human thyroid hormone receptor. Molecular Endocrinology 14 718-732.

Bachmair A, Finley D \& Varshavsky A 1986 In vivo half-life of a protein is a function of its amino-terminal residue. Science 234 179-186.

Butt TR \& Chen DJ 1999 Reconstruction of mammalian nuclear receptor function in Saccharomyces cerevisiae. In Manual of Industrial Microbiology and Biotechnology, 2nd edition, Section V, pp 527-538. Eds J Davies \& C Harshberger. Washington, DC: American Society for Microbiology.

Butt TR \& Walfish PG 1996 Human nuclear receptor heterodimers: opportunities for detecting targets of transcriptional regulation using yeast. Gene Expression 5 255-268.

Cavailles V, Dauvois S, L'Horset F, Lopez G, Hoare S, Kushner PJ \& Parker MG 1995 Nuclear factor RIP140 modulates transcriptional activation by the estrogen receptor. EMBO Fournal 14 3741-3751.

Chen JD, Umesono K \& Evans RM 1996 SMRT isoforms mediate repression and anti-repression of nuclear receptor heterodimers. PNAS 93 7567-7571.

Cho WL, Kapitskaya MZ \& Raikhel AS 1995 Mosquito ecdysteroid receptor: analysis of the cDNA and expression during vitellogenesis. Insect Biochemistry and Molecular Biology 25 19-27.

Chrivia JC, Kwok RP, Lamb N, Hagiwara M, Montminy MR \& Goodman RH 1993 Phosphorylated CREB binds specifically to the nuclear protein CBP. Nature 365 $855-859$.

Dela Cruz F \& Mak P 1997 Drosophila ecdysone receptor functions as a constitutive activator in yeast. Fournal of Steroid Biochemistry and Molecular Biology 62 353-359.

Dela Cruz FE, Kirsch DR \& Heinrich JN 2000 Transcriptional activity of Drosophila melanogaster ecdysone receptor isoforms and ultraspiracle in Saccharomyces cerevisiae. Fournal of Molecular Endocrinology 24 183-191.

Dhadialla TS, Carlson GR \& Le DP 1998 New insecticides with ecdysteroidal and juvenile hormone activity. Annual Review of Entomology 43 545-569.

El-Tanani MK \& Green CD 1997 Interaction between estradiol and growth factors in the regulation of specific gene expression in MCF-7 human breast cancer cells. Fournal of Steroid Biochemistry and Molecular Biology 60 269-276.

Fujiwara H, Jindra M, Newitt R, Palli SR, Hiruma K \& Riddiford LM 1995 Cloning of an ecdysone receptor homolog from Manduca sexta and the developmental profile of its mRNA in wings. Insect Biochemistry and Molecular Biology 25 845-856.

Gietz D, St Jean A, Woods RA \& Schiestl RH 1992 Improved method for high efficiency transformation of intact yeast cells. Nucleic Acids Research 201425.
Graumann K, Wittliff JL, Raffelsberger W, Miles L, Jungbauer A \& Butt TR 1996 Structural and functional analysis of N-terminal point mutants of the human estrogen receptor. Fournal of Steroid Biochemistry and Molecular Biology 57 293-300.

Hatzivassiliou E, Cardot P, Zannis VI \& Mitsialis SA 1997 Ultraspiracle, a Drosophila retinoic X receptor alpha homologue, can mobilize the human thyroid hormone receptor to transactivate a human promoter. Biochemistry 36 9221-9231.

Henrich VC, Sliter TJ, Lubahn DB, MacIntyre A \& Gilbert LI 1990 A steroid/thyroid hormone receptor superfamily member in Drosophila melanogaster that shares extensive sequence similarity with a mammalian homologue. Nucleic Acids Research 18 4143-4148.

Hong H, Kohli K, Trivedi A, Johnson DL \& Stallcup MR 1996 GRIP1, a novel mouse protein that serves as a transcriptional coactivator in yeast for the hormone binding domains of steroid receptors. PNAS 93 4948-4952.

Horlein AJ, Naar AM, Heinzel T, Torchia J, Gloss B, Kurokawa R, Ryan A, Kamei Y, Soderstrom M, Glass CK et al. 1995 Ligand-independent repression by the thyroid hormone receptor mediated by a nuclear receptor co-repressor. Nature 377 397-404.

Ikonen T, Palvimo JJ \& Janne OA 1997 Interaction between the amino- and carboxyl-terminal regions of the rat androgen receptor modulates transcriptional activity and is influenced by nuclear receptor coactivators. Fournal of Biological Chemistry 272 29821-29828.

Imhof MO, Rusconi S \& Lezzi M 1993 Cloning of a Chironomus tentans cDNA encoding a protein $(\mathrm{cEcRH})$ homologous to the Drosophila melanogaster ecdysteroid receptor $(\mathrm{dEcR})$. Insect Biochemistry and Molecular Biology $23115-124$.

Jindra M, Malone F, Hiruma K \& Riddiford LM 1996 Developmental profiles and ecdysteroid regulation of the mRNAs for two ecdysone receptor isoforms in the epidermis and wings of the tobacco hornworm, Manduca sexta. Develomental Biology 180 258-272.

Jindra M, Huang JY, Malone F, Asahina M \& Riddiford LM 1997 Identification and mRNA developmental profiles of two ultraspiracle isoforms in the epidermis and wings of Manduca sexta. Insect Molecular Biology 6 41-53.

Jones G \& Sharp PA 1997 Ultraspiracle: an invertebrate nuclear receptor for juvenile hormones. PNAS 94 13499-13503.

Joyeux A, Cavailles V, Balaguer P \& Nicolas JC 1997 RIP 140 enhances nuclear receptor-dependent transcription in vivo in yeast. Molecular Endocrinology 11 193-202.

Kamimura M, Tomita S \& Fujiwara H 1996 Molecular cloning of an ecdysone receptor (B1 isoform) homologue from the silkworm, Bombyx mori, and its mRNA expression during wing disc development. Comparative Biochemistry and Physiology: B-Biochemistry and Molecular Biology 113 341-347.

Kamimura M, Tomita S, Kiuchi M \& Fujiwara H 1997 Tissue-specific and stage-specific expression of two silkworm ecdysone receptor isoforms - ecdysteroid-dependent transcription in cultured anterior silk glands. European Fournal of Biochemistry 248 786-793.

Kapitskaya M, Wang S, Cress DE, Dhadialla TS \& Raikhel AS 1996 The mosquito ultraspiracle homologue, a partner of ecdysteroid receptor heterodimer: cloning and characterization of isoforms expressed during vitellogenesis. Molecular and Cellular Endocrinology 121 119-132.

Koelle MR, Talbot WS, Segraves WA, Bender MT, Cherbas P \& Hogness DS 1991 The Drosophila EcR gene encodes an 
ecdysone receptor, a new member of the steroid receptor superfamily. Cell 67 59-77.

Kothapalli R, Palli R, Ladd TR, Sohi SS, Cress D, Dhadialla TS, Tzertzinis G \& Retnakaran A 1995 Cloning and developmental expression of the ecdysone receptor gene from the spruce budworm, Choristoneura fumiferana. Developmental Genetics 17 319-330.

Lan Q \& Riddiford LM 1997 DNA transfection in the ecdysteroid-responsive GV1 cell line from the tobacco hornworm, Manduca sexta. In Vitro Cellular and Developmental Biology. Animal 33 615-621.

Lan Q, Hiruma K, Hu X, Jindra M \& Riddiford LM 1999 Activation of a delayed-early gene encoding MHR3 by the ecdysone receptor heterodimer EcR-B1-USP-1 but not by EcR-B1-USP-2. Molecular and Cellular Biology 19 4897-4906.

Lyttle CR, Damian-Matsumura P, Juul H \& Butt TR 1992 Human estrogen receptor regulation in a yeast model system and studies on receptor agonists and antagonists. Fournal of Steroid Biochemistry and Molecular Biology 42 677-685.

Mak P, Fuernkranz HA, Ge R \& Karathanasis SK 1994 Retinoid X receptor homodimers function as transcriptional activators in yeast. Gene 145 129-133.

Metzger D, White JH \& Chambon P 1988 The human oestrogen receptor functions in yeast. Nature 334 31-36.

Nagy L, Kao HY, Love JD, Li C, Banayo E, Gooch JT, Krishna V, Chatterjee K, Evans RM \& Schwabe JW 1999 Mechanism of corepressor binding and release from nuclear hormone receptors. Genes and Development 13 3209-3216.

Onate SA, Tsai SY, Tsai MJ \& O’Malley BW 1995 Sequence and characterization of a coactivator for the steroid hormone receptor superfamily. Science 270 1354-1357.

Onate SA, Boonyaratanakornkit V, Spencer TE, Tsai SY, Tsai MJ, Edwards DP \& O’Malley BW 1998 The steroid receptor coactivator-1 contains multiple receptor interacting and activation domains that cooperatively enhance the activation function 1 (AF1) and AF2 domains of steroid receptors. Fournal of Biological Chemistry 273 12101-12108.

Oro AE, McKeown M \& Evans RM 1990 Relationship between the product of the Drosophila ultraspiracle locus and the vertebrate retinoid X receptor. Nature 347 298-301.

Perera SC, Palli SR, Ladd TR, Krell PJ \& Retnakaran A 1998 The ultraspiracle gene of the spruce budworm, Choristoneura fumiferana: cloning of cDNA and developmental expression of mRNA. Developmental Genetics 22 169-179.

Perera SC, Sundaram M, Krell PJ, Retnakaran A, Dhadialla TS \& Palli SR 1999 An analysis of ecdysone receptor domains required for heterodimerization with ultraspiracle. Archives of Insect Biochemistry and Physiology 41 61-70.

Retnakaran A, Hiruma K, Palli S \& Riddiford L 1995 Molecular analysis of the mode of action of RH-5992, a lepidopteran-specific, non-steroidal ecdysteroid agonist. Insect Biochemistry and Molecular Biology 25 109-117.

Riddiford L 1993 Hormones and Drosophila development. In The Development of Drosophila Melanogaster, pp 899-939. Eds M Bate \& A Martinez-Arias. New York: Cold Spring Harbor Laboratory.

Riddihough G \& Pelham H 1987 An ecdysone response element in the Drosophila melanogaster. EMBO Fournal 21 181-197.

Sambrook J, Fritsch E \& Maniatis T 1989 Molecular Cloning: A Laboratory Manual. New York: Cold Spring Harbor Laboratory.

Shea MJ, King DL, Conboy MJ, Mariani BD \& Kafatos FC 1990 Proteins that bind to Drosophila chorion cis-regulatory elements: a new $\mathrm{C} 2 \mathrm{H} 2$ zinc finger protein and a $\mathrm{C} 2 \mathrm{C} 2$ steroid receptor-like component. Genes and Development 4 1128-1140.
Sheeler CQ, Dudley MW \& Khan SA 2000 Environmental estrogens induce transcriptionally active estrogen receptor dimers in yeast: activity potentiated by the coactivator RIP140. Environmental Health Perspectives 108 97-103.

Sherman F, Fink G \& Hicks J 1986 Methods in Yeast Genetics. New York: Cold Spring Harbor Laboratory.

Sikorski RS \& Hieter P 1989 A system of shuttle vectors and yeast host strains designed for efficient manipulation of DNA in Saccharomyces cerevisiae. Genetics 122 19-27.

Storici F, Coglievina M \& Bruschi CV 1999 A 2-microm DNA-based marker recycling system for multiple gene disruption in the yeast Saccharomyces cerevisiae. Yeast 15 271-283.

Suhr ST, Gil EB, Senut MC \& Gage FH 1998 High level transactivation by a modified Bombyx ecdysone receptor in mammalian cells without exogenous retinoid $\mathrm{X}$ receptor. PNAS 95 7999-8004.

Swevers L, Drevet JR, Lunke MD \& Iatrou K 1995 The silkmoth homolog of the Drosophila ecdysone receptor (B1 isoform): cloning and analysis of expression during follicular cell differentiation. Insect Biochemistry and Molecular Biology $25857-866$.

Talbot WS, Swyryd EA \& Hogness DS 1993 Drosophila tissues with different metamorphic responses to ecdysone express different ecdysone receptor isoforms. Cell $\mathbf{7 3}$ 1323-1337.

Thomas HE, Stunnenberg HG \& Stewart AF 1993 Heterodimerization of the Drosophila ecdysone receptor with retinoid X receptor and ultraspiracle. Nature 362 471-475.

Thormeyer D, Tenbaum SP, Renkawitz R \& Baniahmad A 1999 EcR interacts with corepressors and harbours an autonomous silencing domain functional in both Drosophila and vertebrate cells. Fournal of Steroid Biochemistry and Molecular Biology 68 163-169.

Thummel CS 1995 From embryogenesis to metamorphosis: the regulation and function of Drosophila nuclear receptor superfamily members. Cell 83 871-877.

Tran HT, Askari HB, Saaban S, Price L, Palli SB, Dhadialla TS, Carlson GR \& Butt TR 2001 Reconstruction of ligand-dependent transactivation of Choristoneura fumiferana ecdysone receptor in yeast. Molecular Endocrinology $\mathbf{1 5}$ 1140-1153.

Tsai CC, Kao HY, Yao TP, McKeown M \& Evans RM 1999 SMRTER, a Drosophila nuclear receptor coregulator, reveals that EcR-mediated repression is critical for development. Molecular Cell 4 175-186.

Tzertzinis G, Malecki A \& Kafatos FC 1994 BmCF1, a Bombyx mori RXR-type receptor related to the Drosophila ultraspiracle. Fournal of Molecular Biology 238 479-486.

Verras M, Mavroidis M, Kokolakis G, Gourzi P, Zacharopoulou A \& Mintzas AC 1999 Cloning and characterization of $\mathrm{CcEcR}$. An ecdysone receptor homolog from the mediterranean fruit fly Ceratitis capitata. European Fournal of Biochemistry 265 798-808.

Vogtli M, Elke C, Imhof MO \& Lezzi M 1998 High level transactivation by the ecdysone receptor complex at the core recognition motif. Nucleic Acids Research 26 2407-2414.

Walfish PG, Yoganathan T, Yang YF, Hong H, Butt TR \& Stallcup MR 1997 Yeast hormone response element assays detect and characterize GRIP1 coactivator-dependent activation of transcription by thyroid and retinoid nuclear receptors. PNAS 94 3697-3702.

Wang SF, Miura K, Miksicek RJ, Segraves WA \& Raikhel AS 1998 DNA binding and transactivation characteristics of the mosquito ecdysone receptor-ultraspiracle complex. Fournal of Biological Chemistry 273 27531-27540. 
Wang SF, Ayer S, Segraves WA, Williams DR \& Raikhel AS $2000 a$ Molecular determinants of differential ligand sensitivities of insect ecdysteroid. Molecular and Cellular Biology 20 3870-3879.

Wang SF, Li C, Zhu J, Miura K, Miksicek RJ \& Raikhel AS $2000 b$ Differential expression and regulation by 20-hydroxyecdysone of mosquito ultraspiracle isoforms. Developmental Biology 218 99-113.

Wilkinson JR \& Towle HC 1997 Identification and characterization of the AF-1 transactivation domain of thyroid hormone receptor beta1. Fournal of Biological Chemistry 272 23824-23832.

Wing K, Slawecki RA \& Carlson GR 1988 RH-5849 a nonsteroidal ecdysone agonist: effect on larval Lepidoptera. Science 241 470-471.
Yao TP, Segraves WA, Oro AE, McKeown M \& Evans RM 1992 Drosophila ultraspiracle modulates ecdysone receptor function via heterodimer formation. Cell 71 63-72.

Yao TP, Forman BM, Jiang Z, Cherbas L, Chen JD, McKeown M, Cherbas P \& Evans RM 1993 Functional ecdysone receptor is the product of EcR and ultraspiracle genes. Nature 366 476-479.

Zhu J, Miura K, Chen L \& Raikhel AS 2000 AHR38, a homolog of NGFI-B, inhibits formation of the functional ecdysteroid receptor in the mosquito Aedes aegypti. EMBO Fournal 19 253-262.

RECEIVED IN FINAL FORM 30 May 2001 ACCEPTED 28 June 2001 